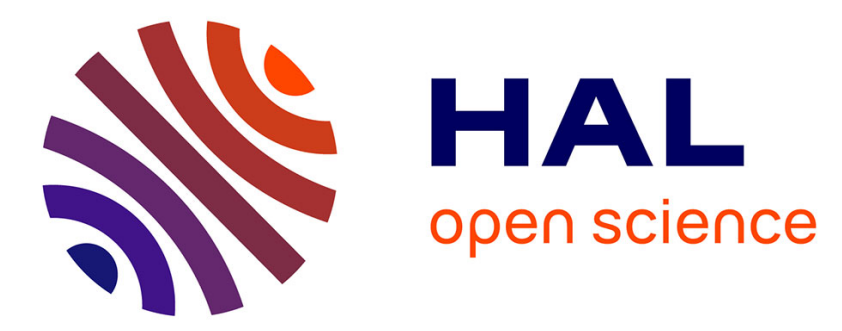

\title{
No-arbitrage macroeconomic determinants of the yield curve
}

\author{
Ruslan Bikbov, Mikhail Chernov
}

\section{To cite this version:}

Ruslan Bikbov, Mikhail Chernov. No-arbitrage macroeconomic determinants of the yield curve. Econometrics, 2010, 159 (1), pp.166. 10.1016/j.jeconom.2010.05.004 . hal-00732517

\section{HAL Id: hal-00732517 https://hal.science/hal-00732517}

Submitted on 15 Sep 2012

HAL is a multi-disciplinary open access archive for the deposit and dissemination of scientific research documents, whether they are published or not. The documents may come from teaching and research institutions in France or abroad, or from public or private research centers.
L'archive ouverte pluridisciplinaire HAL, est destinée au dépôt et à la diffusion de documents scientifiques de niveau recherche, publiés ou non, émanant des établissements d'enseignement et de recherche français ou étrangers, des laboratoires publics ou privés. 


\section{Accepted Manuscript}

No-arbitrage macroeconomic determinants of the yield curve

Ruslan Bikbov, Mikhail Chernov

PII:

S0304-4076(10)00129-6

DOI:

10.1016/j.jeconom.2010.05.004

Reference: ECONOM 3382

To appear in: Journal of Econometrics

Received date: 29 October 2008

Revised date: 8 October 2009

Accepted date: 24 May 2010

Please cite this article as: Bikbov, R., Chernov, M., No-arbitrage macroeconomic determinants of the yield curve. Journal of Econometrics (2010), doi:10.1016/j.jeconom.2010.05.004

This is a PDF file of an unedited manuscript that has been accepted for publication. As a service to our customers we are providing this early version of the manuscript. The manuscript will undergo copyediting, typesetting, and review of the resulting proof before it is published in its final form. Please note that during the production process errors may be discovered which could affect the content, and all legal disclaimers that apply to the journal pertain. 


\title{
No-Arbitrage Macroeconomic Determinants of the Yield Curve
}

\author{
Ruslan Bikbov $^{\mathrm{a}}$, Mikhail Chernov ${ }^{\mathrm{b}, *}$, \\ ${ }^{a}$ Federal Reserve Board, Washington, DC 20551, USA \\ ${ }^{\mathrm{b}}$ London Business School, London School of Economics, and CEPR, London, \\ $N W 14 S A, U K$
}

\begin{abstract}
No-arbitrage macro-finance models use variance decompositions to gauge the extent of association between the macro variables and yields. We show that results generated by this approach are sensitive to the order of variables in the recursive identification scheme. In a four-factor model, one may obtain 18 different sets of answers out of 24 possible. We propose an alternative measure that is based on levels of macro variables as opposed to shocks. We account for the correlation between the macro and latent factors via projection of the latter onto the former. As a result, the association between macro variables and yields can be computed uniquely via an $R^{2}$. Macro variables explain $80 \%$ of the variation in the short rate and $50 \%$ of the slope, and $54 \%$ to $68 \%$ of the term premia.
\end{abstract}

JEL Classification: C13; C22; G12

Keywords: macro-finance models; term structure; variance decomposition; Kalman filter 


\section{Introduction}

The important work of Ang and Piazzesi (2003) extends the traditional no-arbitrage term structure models by modelling jointly the dynamics of yields and nonfinancial variables. The nonfinancial variables chosen by researchers are typically macro variables, so we refer to the models as no-arbitrage macro-finance models. One of the most important issues that the setting allows researchers to address is whether yields are associated with macro variables. This issue could be broken down further. First, is the association between yields and macro variables determined by the monetary policy? There is a strong intuition from the Taylor rule literature that suggests that such macro variables as inflation and real activity should matter for the interest rate, which is the monetary policy instrument. ${ }^{1}$ Second, is the association between yields and macro variables determined by the yield risk premia? It could be that macro variables affect the yields exclusively through the spot interest rate. Finally, regardless of the channel, how large is this association quantitatively? A precise answer to these questions will provide a quantitative benchmark for equilibrium models. If structural implications of these models are to be taken seriously, they should be able to generate, at the very least, the same common variation in yields and macro variables as the benchmark.

\footnotetext{
ऋ We are grateful to the Editor, Ron Gallant, the Associate Editor and the Referee for invaluable comments that helped us to improve the paper. We thank Andrew Ang, Geert Bekaert, Alan Bester, Jean Boivin, Larry Christiano, Pierre Collin-Dufresne, Greg Duffee, Silverio Foresi, Mike Gallmeyer, Rene Garcia, Marc Giannoni, Francisco Gomes, Mike Johannes, Lars Lochstoer, Stijn Van Nieuwerburgh, Tarun Ramadorai, Andrea Roncoroni, Tano Santos, Suresh Sundaresan, Andrea Tambalotti and participants of workshops at Columbia, Bank of Canada, the Federal Reserve Bank of Atlanta, and AFA in Boston, CIREQ-CIRANO Financial Econometrics Conference in Montreal, the CEPR meetings at Gerzensee, Econometric World Congress in London, EFA in Moscow, NYU Stern Five-Star Conference, and seminars at Chicago GSB, Duke, the European Central Bank, the Federal Reserve Board, the Federal Reserve Bank of New York, Goldman Sachs Asset Management, Imperial College, JP Morgan, LBS, LSE, NYU, Princeton and Rice. Chernov acknowledges support of the JP Morgan Chase Academic Outreach program.

* Corresponding author. London Business School, Sussex Place, London, NW1 4SA, United Kingdom. Tel.: +442070008258

Email addresses: ruslan.bikbov@frb.gov (Ruslan Bikbov), mchernov@london.edu (Mikhail Chernov).

1 However, the degree of association is not obvious. In fact, interest rate rules and, therefore, their ingredients, do not matter at all in the absence of frictions, such as price stickiness (e.g., Bils and Klenow, 2004 and Woodford, 2003).
} 
The answers to these questions that are found in the literature are mixed. On the one hand, as is well-known from the term-structure studies using financial variables only, such as Litterman and Scheinkman (1991) or Dai and Singleton (2000), two or three factors are sufficient for capturing 95\%-98\% of the variation in the yield curve. This conclusion suggests a small role for macro variables in a empirically realistic term structure model. On the other hand, because the macro-finance models can be cast in a vector autoregression (VAR) framework, it is natural to quantify the common variation in yields and macro variables via variance decomposition. Studies that follow this route attribute a large fraction ( $40 \%$ to $80 \%$ ) of the unconditional variance in yields to the joint contribution of real activity and inflation, which suggests that macro determinants are very important. ${ }^{2}$

The reason that the two strands of the term-structure literature produce seemingly conflicting results is that the VAR literature uses shocks to macro variables to explain variation in yields, while the work based only on financial data focuses on the levels of variables to explain the same variation. It should be possible to reconcile the two approaches because levels are aggregates of shocks. In this paper, we propose a general procedure that allows us to obtain a correct measurement of the association between yields and macro variables.

A simple example illustrates how such a reconciliation can be achieved. Consider the Ang and Piazzesi (2003) model, where macro variables are independent from the latent variables. The infinite horizon variance decompositions from the VAR representation of the model characterize how much of the unconditional yield variance can be attributed to shocks in each of the macro variables. Our main interest is in disentangling the contribution of the macro and latent variables, rather than in disentangling every single variable. Therefore, we can exploit the independence of the two types of variables (macro and latent) and determine the proportion of the yield variance that can be explained by macro variables simply by computing the corresponding $R^{2}$.

The two approaches will yield the same result under two important conditions. First, the shocks have to be identified correctly. Researchers often employ the recursive identification ${ }^{2}$ For example, Ang et al. (2004), Ang and Piazzesi (2003), Dai and Philippon (2004), Rudebusch and $\mathrm{Wu}(2005)$, among others. 
scheme in the context of reduced-form VARs, such as macro-finance models. There is no guarantee that a particular order of variables will produce the correct identification; hence, it is possible for there to be multiple sets of measures. Second, the VAR has to provide a good fit to the data, in that the variance of yields in the data and in the model should be similar. This is because the computation of variance decomposition does not involve actual data. In contrast, an $R^{2}$ measures how much of the actual variance is explained by a model.

The coefficient of determination, $R^{2}$, appears to be a more robust measure because it relies on observed macro variables as opposed to unobserved shocks and, therefore, is not subject to assumptions about identification. We emphasize that $R^{2}$ will not only be a unique measure of association between macro variables and yields; it will also be a correct measure. However, this measure cannot be implemented if macro and latent variables are correlated with each other. This would be the case if, for example, there is feedback from the financial sector into the real economy.

We resolve this problem by exploiting the special nature of latent variables. Latent variables do not have any macro interpretation. As Dai and Singleton (2000) point out, these factors can be re-parameterized, or rotated, in a number of different ways without changing the value of yields. Thus, latent factors serve no purpose other than to fit the data well. These observations lead us to propose a novel procedure that allows to express the traditional latent factors via macro variables and new "residual" latent variables that are conditionally orthogonal to the macro ones. As a result, we disentangle the contribution of the macro and the new latent variables to the variation of yields. Now we can implement the $R^{2}$ that we advocated above.

The key to our procedure is a conditional model-based projection of the latent variables onto the macro ones. The projection effectively breaks the latent factors down into (i) a component that consists of the current and past macro variables, and (ii) new "projection residual" latent variables that are conditionally orthogonal to the entire history of macroeconomic variables. By construction, the variance of the new latent factors is minimized and the variance of the macro component is maximized. The spot interest rate becomes a linear function of macro 
variables, their lags, and a set of new latent factors. ${ }^{3}$

The empirical implementation of our procedure is based on a four-factor Gaussian model with two latent factors and inflation and real activity acting as observable factors. To estimate the model, we use a panel of eight yields ranging from three months to 10 years, with inflation and real activity observed at a monthly frequency from 1970 to 2002. We provide diagnostics that indicate that the model fits the data reasonably well.

We document that the variance decomposition results are extremely sensitive to the order of the model factors chosen for the recursive identification. For example, the fraction of the interest rate variance that is explained by the joint shocks to inflation and real activity can vary between $36 \%$ and $77 \%$ depending on the assumed order of the factors. Similarly, the fraction of the explained slope variance varies between $25 \%$ and $81 \% .{ }^{4}$ In particular, the standard order of the variables, that is, macro variables followed by latent variables, produces $52 \%$ for the short interest rate and $37 \%$ for the slope. Our procedure produces a unique set of explained variances precisely because it is based on observed macro variables. In particular, $80 \%$ of the variation in the interest rate and $52 \%$ of the variation in the slope can be explained by inflation and real activity. A similar set of figures ( $76 \%$ and $59 \%$, respectively) is obtained via the variance decomposition if the order of the variables is real activity followed by latent variables, which are in turn followed by inflation. Clearly, there is no way of knowing this "correct" order of shocks in the context of a reduced-from model.

The point that variance decomposition results are sensitive to the order of variables in the recursive identification scheme is not new in itself. Nevertheless, given the popularity of the identification scheme in the macro-finance literature on the term structure, it is worth revisiting. The uncovered magnitudes of disagreement between the variance decompositions based on different orders of variables seem to warrant a consideration of an alternative approach. To be sure, our projection-based procedure is not a silver bullet. It cannot replace

\footnotetext{
${ }^{3}$ Our lag structure is not arbitrary: recursive projection formulas imply reliance on all lags and the loadings on these lags are optimal, due to the fact that they are selected to minimize the variance of the residuals.

${ }^{4}$ We measure the slope of the yield curve as the difference between the 10-year and three-month yields.
} 
variance decompositions because it can only work in the presence of latent variables and has no causal implications. We view our procedure as a useful complement to the toolkit of a researcher studying VARs that are related to the yield curve.

We further illustrate the usefulness of our procedure by using it to address the issues raised at the beginning of the introduction. ${ }^{5}$ First, we investigate how much of the short rate variation can be explained by the two macro variables, as opposed to by the latent factors. It seems natural to start with the null hypothesis that macro variables do not contribute to explaining the short rate, given the excellent explanatory power of latent factor models that we discussed earlier. As we show, this hypothesis places restrictions on various parameters that control the dynamics and cross-section of the yield curve. A number of previous studies have simply imposed these restrictions, thereby limiting the ability of yields to vary with the macroeconomy. We test these restrictions in terms of the size of $R^{2}$ that can be explained by macro variables and confirm that macro variables are extremely important. Our projectionbased approach can explain $80 \%$ of the short rate variation, using inflation, real activity, and their lags exclusively as the basis.

Second, we investigate whether macroeconomic variables are useful for explaining bond term premia and not just short-term interest rates. ${ }^{6}$ Our approach enables us to determine that inflation and real activity risk premia are both significant factors, which jointly explain $54 \%$ to $68 \%$ of the variation in the term premia, depending on a bond's maturity. In the context of time-series analysis, the contribution of inflation is countercyclical. It is also the most important contributor with respect to the magnitude and volatility of term premia.

Third, we focus on the nature of the residual latent variables. In order to gauge the relationship between additional macro variables and our latent factors, we correlate them with two measures of liquidity (AAA credit spread and the growth rate of the money zero maturity

\footnotetext{
$\overline{5}$ More recent works by Chernov and Mueller (2008) and Mueller (2008) provide further examples of applications of our methodology.

${ }^{6}$ In fact, there is currently a debate in the literature over this point. Ang et al. (2004) and Ludvigson and $\mathrm{Ng}$ (2009) find that inflation and real activity measures play an important role in explaining bond risk premia. In contrast, Duffee (2007) concludes that macro variables make a minimal contribution to the term premia.
} 
(MZM), which is a measure of money supply) and a measure of the budget deficit (public government debt growth). The correlations are strong, but not overwhelming. We therefore conclude that it is more appropriate to use latent variables to model shocks. The shock associated with liquidity measures contributes to the variation in short interest rate, almost completely accounting for the part left unexplained by the macro variables. We interpret this factor as an exogenous policy shock, that is, occasional reactions of the short interest rate to information beyond that contained in real activity and inflation. The shocks correlated with the budget deficit make the largest contribution to that portion of the slope variation that is left unexplained by macro variables. We interpret this factor as an exogenous non-policy shock.

Clearly, our paper is related to the growing literature on macro-finance yield curve modelling. ${ }^{7}$ Our work, while having a different focus, is particularly close to the concurrent study by Ang et al. (2004) (ADP henceforth). ADP use a macro-finance model to show that one and the same interest rule can be interpreted in three distinct ways. Given that it is based on additional assumptions such as the forecasting horizon and whether exogenous shocks depend on current or past innovations in the state variables, the interest rule can be seen as either simple, forward-looking, or backward-looking. Their approach applies to a model with only one latent factor. In their empirical work, ADP use variance decompositions to gauge the degree of association between the macro variables and yields. Our projectionbased approach implies a backward-looking interest rate rule and so appears to be similar to their work. However, there are important differences in the focus and approach. First, our procedure applies regardless of the number of latent factors in the model. Second, we do not focus on the interest rate rules per se, precisely because structural interpretation requires additional assumptions. Third, regardless of the assumed interest rate rule, variance decompositions will be the same and our focus is on providing a robust alternative to such a computation.

7 This literature includes Ang and Piazzesi (2003), Bekaert et al. (2010), Dai and Philippon (2004), Duffee (2006), Diebold et al. (2006), Gallmeyer et al. (2005), Hördahl et al. (2006), Law (2004), Rudebusch and $\mathrm{Wu}$ (2005), among others. 
The remainder of this paper is organized into five sections and two appendixes. Section 2 summarizes the macro-finance framework. Section 3 motivates and describes the proposed methodology. Section 4 discusses the estimation strategy. Section 5 presents our findings. The final section concludes. Technical details are presented in the appendices.

\section{The Model}

\subsection{State variables}

Following Ang and Piazzesi (2003) (AP henceforth), we assume that the state of the economy is captured by the vector $z_{t}=\left(m_{t}^{\prime}, x_{t}^{\prime}\right)^{\prime}$. In particular, the vector of macroeconomic variables $m_{t}$ is equal to $\left(g_{t}, \pi_{t}\right)^{\prime}$, where $g_{t}$ and $\pi_{t}$ are monthly real activity and the inflation rate, respectively. The remaining factors $x_{t}$ are latent. The latent factors may contain the lags of $m_{t}$, other macro variables, or other unknown variables. Importantly, the vector $z_{t}$ fully reflects all available information at time $t$; so, for instance, one need not consider lags of $x_{t}$.

We assume that the state vector $z_{t}$ follows a VAR(1) process:

$$
\begin{aligned}
z_{t} & =\mu+\Phi z_{t-1}+\Sigma \epsilon_{t} \\
& =\left[\begin{array}{l}
\mu^{m} \\
\mu^{x}
\end{array}\right]+\left[\begin{array}{cc}
\Phi^{m m} & \Phi^{m x} \\
\Phi^{x m} & \Phi^{x x}
\end{array}\right]\left[\begin{array}{l}
m_{t-1} \\
x_{t-1}
\end{array}\right]+\left[\begin{array}{cc}
\Sigma^{m m} & \Sigma^{m x} \\
\Sigma^{x m} & \Sigma^{x x}
\end{array}\right]\left[\begin{array}{c}
\epsilon_{t}^{m} \\
\epsilon_{t}^{x}
\end{array}\right],
\end{aligned}
$$

where $\epsilon_{t} \sim N(0, I)$. We denote the vector of parameters that control the dynamics of state by $\Theta=(\mu, \Phi, \Sigma)$. The block representation will be useful for later discussions. 


\subsection{No-Arbitrage Bond Valuation}

It is customary in the term structure literature to specify the interest rate as a linear function of the state variables (e.g., Dai and Singleton, 2000, or AP):

$$
r_{t}=\delta_{0}+\delta_{z}^{\prime} z_{t}
$$

The specification of the state variables, combined with the interest rate specification in (2), allows us to complete the usual affine no-arbitrage framework by specifying the stochastic discount factor $\xi_{t}$ :

$$
\log \xi_{t}=-r_{t-1}-\frac{1}{2} \Lambda_{t-1}^{\prime} \Lambda_{t-1}-\Lambda_{t-1} \epsilon_{t}
$$

where the market prices of risk follow the essentially-affine specification (Duffee, 2002)

$$
\Lambda_{t}=\Lambda_{0}+\Lambda_{z} z_{t}
$$

Therefore, yields on zero-coupon bonds are linear in the state variables:

$$
\begin{aligned}
y_{t}(\tau) & =-\frac{1}{\tau} \log E_{t}\left(\prod_{s=t+1}^{t+\tau} \xi_{s}\right)=a^{Q}(\tau)+b^{Q}(\tau)^{\prime} z_{t} \\
& \equiv a^{P}(\tau)+b^{P}(\tau)^{\prime} z_{t} \quad+a^{T P}(\tau)+b^{T P}(\tau)^{\prime} z_{t},
\end{aligned}
$$

where $\tau$ is the respective maturity, and $a^{Q}$ and $b^{Q}$ solve recursive equations with boundary conditions $a^{Q}(1)=\delta_{0}$, and $b^{Q}(1)=\delta_{z}$ (see, e.g., Backus et al., 1999). In particular, the onemonth yield coincides with the short rate, $y_{t}(1)=r_{t}$. The last line breaks the yields down into the expectations of the future short rates and the term premium. The former component is equal to the usual factor loadings computed under the assumption of zero market prices of risk. 


\section{Are variations in the yield curve related to the macroeconomy?}

\subsection{What does this question really mean?}

Macro-finance models of the yield curve have the potential to provide a direct answer to the question "Are variations in the yield curve related to the macroeconomy?" However, a reader might think that the question has already been answered by earlier studies. Whether or not this is true will depend on exactly what one means when posing the question.

One way to answer this question is by investigating whether shocks to macro variables are associated with the yields. The variance decomposition framework developed in the VAR literature is ideally suited for this task. However, this approach has its shortcomings. In particular, the approach characterizes the impact of fitted shocks on fitted yields, thereby reflecting the relationships that exist in a model rather than in the data. Moreover, it is difficult to settle on a shock orthogonalization scheme in the absence of any structural identification.

Another way to answer the question is by quantifying the degree to which yields vary along with levels of macro factors: that is, by computing the $R^{2}$ statistic. This measure does not tell us anything about causality or dynamics of the relationship, but it does provide a robust measure of the covariance between observed yields and macro variables.

Levels of macro variables are simple aggregates of the macro shocks. Therefore, it must be the case that, perhaps under certain conditions, the two approaches provide the same answer. We claim that this will happen if the shocks are identified correctly and a model fits the data perfectly. Clearly, both requirements highlight the shortcomings of the variance decomposition approach that were highlighted earlier. A simple example illustrates why the two approaches would coincide in this case.

Suppose that $z_{t}=\left(\pi_{t}, x_{t}\right)^{\prime}$ follows a $\operatorname{VAR}(1)$ process as before and that the two state variables are independent from each other, that is, matrices $\Phi$ and $\Sigma$ in (1)-(1) are diagonal. Further, 
assume that the spot interest rate is simply a sum of the two state variables:

$$
r_{t}=\pi_{t}+x_{t}
$$

In this case, the variance of the interest rate explained by the variation in inflation is simply

$$
R_{\pi}^{2}\left(r_{t}\right)=\frac{\operatorname{var}\left(\pi_{t}\right)}{\operatorname{var}\left(r_{t}\right)}
$$

Here $\operatorname{var}\left(r_{t}\right)$ refers to the variance of observed $r_{t}$. Inflation follows an $\operatorname{AR}(1)$ process:

$$
\pi_{t}=\mu^{\pi}+\Phi^{\pi \pi} \pi_{t-1}+\Sigma^{\pi \pi} \epsilon_{t}^{\pi}
$$

and, therefore,

$$
\operatorname{var}\left(\pi_{t}\right)=\frac{\left(\Sigma^{\pi \pi}\right)^{2}}{1-\left(\Phi^{\pi \pi}\right)^{2}}
$$

Alternatively, the state variables (1) can be represented as aggregates of their shocks:

$$
z_{t+s}=\left(\sum_{j=0}^{s-1} \Phi^{j}\right) \mu+\Phi^{s} z_{t}+\sum_{j=0}^{s-1}\left(\Phi^{j}\right) \Sigma \epsilon_{t+s-j} .
$$

This representation allows us to construct the decomposition of the variance of the error when forecasting $z_{t+s}$ (see, e.g., Hamilton, 1994, for details):

$$
\operatorname{MSE}\left(\hat{z}_{t+s \mid t}\right)=\sum_{j=0}^{s-1}\left(\Phi^{j}\right) \Sigma \Sigma^{\prime}\left(\Phi^{j}\right)^{\prime}=\operatorname{diag}\left(\left(\Sigma^{\pi \pi}\right)^{2} \sum_{j=0}^{s-1}\left(\Phi^{\pi \pi}\right)^{2 j},\left(\Sigma^{x x}\right)^{2} \sum_{j=0}^{s-1}\left(\Phi^{x x}\right)^{2 j}\right)
$$

where the second equality follows from our assumption that the shocks are identified correctly and $\operatorname{diag}\left(a_{1}, \ldots, a_{n}\right)$ is a diagonal matrix with elements from $a_{1}$ to $a_{n}$ along the diagonal.

The interest rate's forecast is 


$$
\hat{r}_{t+s \mid t}=e_{1}^{\prime} \hat{z}_{t+s \mid t}+e_{2}^{\prime} \hat{z}_{t+s \mid t},
$$

where $e_{k}$ is a vector of zeros with a one in position $k$ and

$$
\operatorname{MSE}\left(\hat{r}_{t+s \mid t}\right)=\left(\Sigma^{\pi \pi}\right)^{2} \sum_{j=0}^{s-1}\left(\Phi^{\pi \pi}\right)^{2 j}+\left(\Sigma^{x x}\right)^{2} \sum_{j=0}^{s-1}\left(\Phi^{x x}\right)^{2 j}
$$

As $s \rightarrow \infty$, the left-hand side of (13) converges to the unconditional variance of the interest rate, $\operatorname{var}\left(r_{t}\right)$, and the right-hand side converges to

$$
\frac{\left(\Sigma^{\pi \pi}\right)^{2}}{1-\left(\Phi^{\pi \pi}\right)^{2}}+\frac{\left(\Sigma^{x x}\right)^{2}}{1-\left(\Phi^{x x}\right)^{2}}
$$

Therefore, the contribution of the inflation shocks to the infinite horizon variance decomposition of $r$ is the same as the $R^{2}$ computed in (7) and (9).

This example is basic, because things are particularly easy for both approaches. The independence of the factors allows us to compute $R^{2}$ and the assumed knowledge of shocks allows us to implement the correct variance decomposition. Further, the $R^{2}$ computed on the basis of the variance decomposition will coincide with $R_{\pi}^{2}$ in (7) because the model is assumed to be a perfect description of the data and, therefore, $M S E\left(\hat{r}_{t+s \mid t}\right)$ converges to the variance of observed $r_{t}$. However, in practice, the factors could be correlated and the shocks cannot be identified without additional assumptions. We do not know how to obtain a unique identification for shocks without imposing some structure. So, it is not clear how to implement variance decomposition in a robust way. Moreover, we show in the subsequent empirical analysis that the variance decomposition results are extremely sensitive to the order of variables if the recursive identification scheme is implemented. Finally, in practice, $R_{\pi}^{2}$ in (7) will still measure the extent of common variation between macro variables and yields in the data, while $R^{2}$ computed on the basis of the variance decomposition will measure common variation in the model. For this reason, we argue that $R^{2}$ should be used to circumvent the shock identification problem. In the next section, we discuss how our projection approach allows to resolve the problem of how to compute $R^{2}$ when factors are correlated. 


\subsection{A new approach}

Given the standard macro-finance framework, we can define the new latent factors as factors that are orthogonal to the entire history of the macro variables, which we denote by $m^{t}=\left\{m_{t}, m_{t-1}, \ldots, m_{0}\right\}$. We compute these latent factors via the projection procedure. Specifically, the residual latent factors $f_{t}$ are computed via

$$
f_{t}=x_{t}-\hat{x}\left(m^{t}\right),
$$

where $\hat{x}\left(m^{t}\right)$ denotes the linear projection of $x_{t}$ onto $m^{t}$.

In our setting, the linear projection maximizes the explanatory power of $m^{t}$ with respect to the original latent factors $x_{t}$. Therefore, our procedure ensures that we explain as much of $x_{t}$ with $m^{t}$ as possible. This is a useful property of our approach because it allows us to gauge the entire contribution of the macro factors to any linear function of the latent factors $x_{t}$.

By construction, the linear projection $\hat{x}$ has a simple functional form that fits nicely into the overall linear structure of the model. It could be thought of as having a VAR structure,

$$
\hat{x}\left(m^{t}\right)=c(\Theta)+\sum_{j=0}^{t} c_{t-j}(\Theta) m_{t-j},
$$

where the matrices $c$ are functions of parameters $\Theta$ that control the dynamics of the state variables $z_{t}$. The dependence of the coefficients $c$ on the model parameters allows us to avoid overparameterization, which is a problem in all multiple-lag studies. Appendix A provides the details of the procedure.

This decomposition allows to represent the spot interest rate as:

$$
r_{t}=\delta_{0}+\delta_{m}^{\prime} m_{t}+\delta_{x}^{\prime} x_{t}=\delta_{0}+\delta_{m}^{\prime} m_{t}+\delta_{x}^{\prime} \hat{x}\left(m^{t}\right)+\delta_{x}^{\prime} f_{t} .
$$

The linear dependence of the projection (16) on the lags of macro variables entails that the 
new representation of the interest rate retains all the attractive qualities of the usual affine specifications. As a result of the projection procedure, the interest rate is expressed as the sum of two, macro and latent, conditionally orthogonal components. The macro component may be interpreted as a backward-looking interest rate rule. The new latent factors $f_{t}$ have a residual role in this setup. They could be related to other observable variables; however, by construction, they will not be related to $m_{t}$. Therefore, one can explore the nature of these variables without the risk of confusing them with $m_{t}$. Section 5.4 provides further discussion of whether such interpretation is reasonable.

It is now easy to determine how much of the variation in the interest rate is explained by the macro variables. Assuming the model parameters are known, one simply has to compute the squared correlation between the observable interest rate and the observable macro-component of the interest rate rule (17). Moreover, the answer to this question will be complete in the sense that, under the null hypothesis that a model is correct, it is not possible for the variables $m_{t}$ to explain more.

The same logic applies to risk premia and yields in (4) and (5), respectively. They can be equivalently expressed via the history of macro variables $m^{t}$ and residuals $f_{t}$ based on (15) and (16). Therefore, our procedure allows us to think in terms of observable macro variables regarding not only the interest rate, but the risk premia as well. As noted above, because of the linear structure, the projection procedure maximizes the explanatory power of macro variables $m$ with respect to the risk premia as well. Thus, as in the case of the short interest rate, we can easily determine the contribution of the macro-component to the variation of yields and risk premia.

\subsection{Addressing the question using our methodology}

We now return to the question "Are variations in the yield curve related to the macroeconomy?" and describe the ideas presented above more formally. In a manner similar to the block representation of the state variables in (1), we represent the risk premia in a block 
form:

$$
\Lambda_{t}=\Lambda_{0}+\left[\begin{array}{cc}
\Lambda^{m m} & \Lambda^{m x} \\
\Lambda^{x m} & \Lambda^{x x}
\end{array}\right]\left[\begin{array}{l}
m_{t} \\
x_{t}
\end{array}\right]
$$

Common variation in yields and macro variables may occur via two channels. First, the spot interest rate may vary along with macro variables. This will happen if the macro variables affect the spot rate directly, that is, if $\delta_{m} \neq 0$ in (17). There may also be an indirect effect when $\delta_{x} \neq 0$ in (17), if macro variables are correlated with latent variables, that is, if the restriction $\Phi^{m x}=\Phi^{x m}=\Sigma^{m x}=\Sigma^{x m}=0$ in (1) is violated. We will refer to this restriction as $R P$ (restriction under the $P$ measure).

Second, the yield may covary with macro variables via the risk premia channel. Macro variables affect the premia directly if the restriction $\Lambda^{m m}=\Lambda^{x m}=0$ is violated. We will refer to this condition as $R Q \mathrm{I}$ (restriction I under the $Q$ measure). Macro factors will also affect the risk premia if the restrictions $\Lambda^{m x}=\Lambda^{x x}=0(R Q I I)$ and $R P$ are both violated.

Testing these restrictions will tell us if there is any relationship between yields and macro variables. However, such testing will not suffice to assess the magnitude of the relationship. To assess the magnitude, one has to separate all the macro terms that enter into the spot rate either directly or indirectly (via correlations with macro variables). Our projection procedure achieves this goal.

Specifically, we compute the "theoretical $R^{2}$ " statistic as a simple measure of the relationship magnitude. In contrast with the regular $R^{2}$, which can be considered a by-product of OLS estimation, our measure is computed using the model parameters as a basis. Because the latent factors $f_{t}$ are orthogonal to the history of the macro variables $m^{t}$, it is straightforward to attribute the correct amounts of explanatory power to the latent factors and macro factors.

We compute the fraction of interest rate variance explained by the macro variables as 


$$
R_{m}^{2}\left(r_{t}\right)=\frac{\operatorname{var}\left(\delta_{m}^{\prime} m_{t}+\delta_{x}^{\prime} \hat{x}\left(m^{t}\right)\right)}{\operatorname{var}\left(r_{t}\right)}
$$

where the numerator is computed on basis of the estimated model and the denominator is computed from the data. We can compute similar measures of fit for the yields. For example, macro variables explain

$$
\left.R_{m}^{2}\left(y_{t}(\tau)\right)\right)=\frac{\operatorname{var}\left(b_{m}^{Q}(\tau)^{\prime} m_{t}+b_{x}^{Q}(\tau)^{\prime} \hat{x}\left(m^{t}\right)\right)}{\operatorname{var}\left(y_{t}(\tau)\right)}
$$

of the yield with maturity $\tau$. We can also construct such measures for any linear combination of yields, e.g., the slope, and for the term premia. For instance, for the term premium,

$$
R_{m}^{2}(T P(\tau))=\frac{\operatorname{var}\left(b_{m}^{T P}(\tau)^{\prime} m_{t}+b_{x}^{T P}(\tau)^{\prime} \hat{x}\left(m^{t}\right)\right)}{\operatorname{var}\left(b^{T P}(\tau)^{\prime} z_{t}\right)}
$$

We can formally test the restrictions $R P, R Q$ and others using standard tools such as the Wald test. However, tracing out the web of all possible restrictions can be daunting. As an alternative, we compute the $R^{2}$ from a model, rather than from OLS. This allows us to assess the joint significance of the restrictions in a more intuitive fashion. For example, if macro variables are not related to the short rate, that is if $\delta_{m}=0$ and $R P$ holds, then $R_{m}^{2}\left(r_{t}\right)$ will be equal to zero. Likewise, if $R P$ and $R Q$ I hold, then $R_{m}^{2}(T P(\tau))$ will be zero. Thus, computing the theoretical $R^{2}$ allows us to simultaneously test whether variations in financial variables are related to variations in macro variables and to quantify the strength of the relationship.

Finally, as a diagnostic of the overall model fit, we can compute the fraction of yield variance explained by the full model. For example, for the short rate we compute

$$
R_{z}^{2}\left(r_{t}\right)=\frac{\operatorname{var}\left(\delta_{z}^{\prime} z_{t}\right)}{\operatorname{var}\left(r_{t}\right)}
$$


We use parametric bootstrapping to compute confidence bounds on $R^{2}$. Specifically, we start by simulating 1000 paths from the model using the estimated parameters. Next, we re-estimate the model along each path and compute $R^{2}$ by using the estimated parameters as a basis. To the best of our knowledge, our study is the first to evaluate the statistical significance of such measures.

\subsection{VAR and No-Arbitrage Models}

It is instructive to understand the relation between macroeconomic VARs and no-arbitrage approaches. We will use the following VAR considered in Evans and Marshall (2002) as a reference in our discussion:

$$
\left[\begin{array}{l}
m_{t} \\
y_{t}
\end{array}\right]=\left[\begin{array}{l}
\mu^{m} \\
\mu^{y}
\end{array}\right]+\left[\begin{array}{cc}
\Phi^{m m}(L) & 0 \\
\Phi^{y m}(L) & \Phi^{y y}(L)
\end{array}\right]\left[\begin{array}{l}
m_{t-1} \\
y_{t-1}
\end{array}\right]+\left[\begin{array}{cc}
\Sigma^{m m} & 0 \\
\Sigma^{y m} & \Sigma^{y y}
\end{array}\right]\left[\begin{array}{c}
\epsilon_{t}^{m} \\
\epsilon_{t}^{y}
\end{array}\right]
$$

where $y_{t}$ denotes a vector of yields $y_{t}(\tau)$ with various maturities $\tau$. $\Phi^{*}(L)$ denotes matrix polynomials in the lag operator $L$. The number of lags is typically selected based on an information criterion or specified in an ad-hoc fashion related to the data frequency; i.e., 12 lags are used for monthly data. The blocks of zeroes imply exogeneity of macro variables with respect to the yields.

In this setting, $\epsilon_{t}^{m}$ is interpreted as a vector of structural macroeconomic shocks. The elements of $\epsilon_{t}^{y}$ are yield shocks. The identification scheme for $\Sigma^{m m}, \Sigma^{y m}$, and $\Sigma^{y y}$ will determine the interpretation of the shocks' impact on the variables of interest (impulse response functions). The VAR specification in (23) makes no attempt to connect yields and macro variables in a structural way. Hence there is no particular relationship between the elements of $\Sigma^{m m}$ and $\Sigma^{y m}$, or between $\epsilon_{t}^{m}$ and $\epsilon_{t}^{y}$.

We can express the relationships between the yields and state variables in our no-arbitrage model as a VAR as well, 


$$
\left[\begin{array}{c}
z_{t} \\
y_{t}
\end{array}\right]=\left[\begin{array}{c}
\mu \\
a^{Q}+b^{Q^{\prime}} \mu
\end{array}\right]+\left[\begin{array}{rr}
\Phi & 0 \\
b^{Q^{\prime}} \Phi & 0
\end{array}\right]\left[\begin{array}{l}
z_{t-1} \\
y_{t-1}
\end{array}\right]+\left[\begin{array}{cc}
\Sigma & 0 \\
b^{Q^{\prime}} \Sigma & 0
\end{array}\right]\left[\begin{array}{l}
\epsilon_{t} \\
\omega_{t}
\end{array}\right]
$$

The no-arbitrage requirement imposes tight restrictions on the VAR specification. In particular, a researcher has to be concerned with an identifying scheme for matrix $\Sigma$ only, which does not change with the number of yields considered. This is in sharp contrast to the macro VAR specification where the three matrices $\Sigma^{m m}, \Sigma^{y m}$, and $\Sigma^{y y}$ have to be considered, and the latter two grow with the number of yields. This effect of the no-arbitrage models is well understood.

However, one of the criticisms of the no-arbitrage approach in the macro literature is that, despite well-motivated identification assumptions, these models explain much less of the variation in the yields via the macro factors than do the macro VARs (see, e.g., Evans and Marshall, 2002). We address this critique by showing that without our decomposition in (17), the macro variables are not given a fair chance to explain the term structure.

In addition to resolving this problem, we establish the optimal lag weights in the VAR (23) that are implied by the term structure model. Consequently, all optimal weights are computed from a small set of the parameters of our model which determine the dynamics of the state $z$. A macro VAR structure does not allow for this feature; the weights on lagged variables are determined by the best unconditional fit and can lead to parameter proliferation.

Appendix A shows that the no-arbitrage VAR (24) can be combined with the projectionbased decomposition relationships (17) to yield:

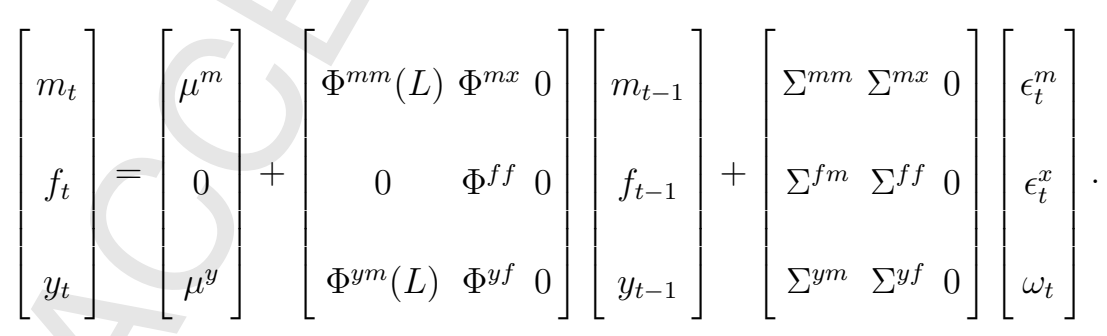


This is a stylized representation; in order to focus on the most important issues, we do not reproduce full expressions for some matrices (e.g., $\Phi^{y m}(L)$ ). We can now develop a better appreciation of the no-arbitrage models by comparing this representation to (23).

At a first glance, the expression in (25) still appears to be more restrictive as compared to the macro VAR specification; many elements of the matrices are restricted to zero, and blocks associated with factor $f$, such as $\Phi^{y f}$, do not have lags. A closer inspection of (25) reveals that if we restrict the block $\Phi^{f f}$ to zero, we will obtain a specification that is qualitatively similar to the macro VAR (23). In this case, $f$ will play the role similar to that of the shocks $\epsilon^{y}$. Hence, we do not lose much flexibility by imposing the no-arbitrage restrictions imposed on the matrices $\Phi^{m m}(L)$ and $\Phi^{y m}(L)$, in particular.

Thus, the specific critique of Evans and Marshall (2002) applies to the particular independence assumptions of AP, rather than the no-arbitrage restrictions. By imposing independence between the latent and factor variables, AP effectively assume that matrices $\Phi^{m x}$, $\Phi^{x m}$, and $\Sigma^{x m}$ in (1) are equal to zero. These restrictions imply, among other things, that $L=0$ in (25).

\subsection{Relationship between the variance decomposition and $R^{2}$}

In earlier sections, we showed that infinite horizon variance decompositions and $R^{2}$ could be reconciled under certain unrealistic assumptions, such as the unique identification of shocks. It is natural to wonder whether anything can be said about the two approaches in more practically relevant situations such as ours. As we emphasized earlier, the $R^{2}$ approach provides a correct determination of the variance in yields that is explained by macro variables. The interesting question is whether we can use these numbers to say something about the variance decompositions.

One approach would be to see whether there is a particular order of state variables in the recursive identification scheme that yields the same answer. If one can find such a match, does it imply that this is the "correct" order in which shocks should be identified? It is 
difficult to say, because the recursive scheme is just one way to identify shocks.

Assuming that this is the only approach to identifying shocks, can one use the $R^{2}$ to infer the order of the variables? The $R^{2}$ approach determines correctly the collective contribution of macro variables when latent variables are present. Our procedure is not applicable when one wants to determine the contribution of a single macro variable, or, similarly, if all state variables are observable. In contrast, the variance decomposition disentangles the contribution of every single variable. Therefore, in principle, there could be multiple decompositions arising from various orderings of the state variables that add up to the same total common variation in yields and macro variables. As a result, we surmise that it would be impossible to develop a general method to back out the correct identification of shocks from the $R^{2}$.

\subsection{Related studies}

Many papers use the impulse response approach and directly or indirectly address whether there is any causal connection between macro variables and yields. However, we do not believe that any of these studies quantify the degree of covariation between macro variables and yields. We will now highlight some of the relevant issues by explaining how existing approaches relate to our framework.

Because we are the first to use the projection method, it is clear that previous studies are unable to address the issue of the relationship's magnitude via the $R^{2}$ unless some additional assumptions are made. In fact, most related work in the literature does impose additional restrictions on parameters. Ignoring for the moment the issue of the appropriate number of factors, some of these models could be nested in ours by imposing the restrictions on parameters that are found in formulae used by (1), (17) and (18).

AP focus on the forecasting implications of adding macro variables to a term structure model. Most of their results are therefore based on impulse responses and decompositions of conditional variances of the forecast errors. Nonetheless, they do evaluate the relationship between the spot rate and macro variables by computing the usual $R^{2}$ from an OLS. AP can 
do this because they impose $R P$. However, due to their different focus, they neither test the significance of $R^{2}$ nor investigate correlations of macro factors with longer yields and risk premia.

Diebold et al. (2006) impose $\delta_{m}=0$ and place implicit restrictions on the risk premia, because the yield loadings on macro variables $b_{m}^{Q}(\tau)$ in $(5)$ are required to be equal to zero for any maturity $\tau{ }^{8}$ They test and reject $R P$ using the likelihood ratio and Wald statistics. Because they introduce other restrictions, the macro factors can only affect yields through their correlations with the finance factors. Their rejection of $R P$ might be explained by this effect. Diebold et al. (2006) are interested in impulse responses and variance decompositions, so they do not report any $R^{2}$-type measures.

Duffee (2007), in concurrent work, focuses exclusively on the question of risk premia. The following conditions are imposed: $R P, \delta_{x}=0, \Lambda^{m x} \neq 0$, and $\Lambda^{x x} \neq 0$ (RQII is violated). These restrictions ensure that there is only one way for risk premia to covary with macro factors - by direct entry in equation (18). For this covariation channel to work, $R Q I$ must be invalid. Duffee rejects this restriction, but finds that the degree of covariation, as measured by $R^{2}$, is weak.

This is all the evidence that is available regarding the relationship between macro factors and yields. As we can see, the choice and degree of imposed restrictions varies quite a bit. The purpose of these restrictions is essentially to shut down one or more channels through which yields may covary with macro factors. Clearly, there is room for investigating the issue of covariation between yields and macro variables within a single unifying framework.

\section{Empirical Setup}

In this section, we apply our projection procedure to investigate the role played by macro and residual latent variables in the dynamics of yields and risk premia. To achieve this we

8 Diebold et al. (2006) do not posit a formal term structure model, so it is difficult to translate their restrictions directly into implications for risk premia. 
must specialize our general setup, select appropriate data, and estimate the model. This section provides all the relevant information.

We start with a brief description of the dataset, then explain the estimation methodology.

We conclude by discussing our parameter identification strategy.

\subsection{Data}

We use a monthly time series of macro and bond data from 1970 to 2002. We use the Consumer Price Index (CPI) and the Help Wanted Index (HWI), both taken from FRED, as proxies for the price level and real activity, respectively. ${ }^{9}$ We compute annual log changes in the CPI index to construct our measure of inflation. The differencing frequency is selected to mitigate the noise in month-to-month changes. Because the HWI is of order 100 (its 1987 level is precisely 100), we divide it by 10 to obtain an order of magnitude similar to those of yields and inflation.

Of course, this is not the only possible choice of macro variables. Apart from alternative reported statistics, the literature has suggested that aggregate measures of inflation and real activity may be more appropriate, judging from principal component analysis (see AP, Boivin and Bernanke, 2003, among others). These studies are partly motivated by the presence of significant measurement noise in stand-alone statistics. Although such considerations are important, they are less critical in the context of our study. "True" measures of the real activity and inflation are not required because we are not estimating a structural model. In effect, we assume that market participants react specifically to the CPI and HWI.

We use an unsmoothed Fama-Bliss approximation to the zero-coupon bond prices, considering maturities of three and six months and one, two, three, five, seven, and 10 years. ${ }^{10}$ It

\footnotetext{
9 The index Help Wanted Advertising in Newspapers, which is a leading indicator of real activity, is used by AP and Dai and Philippon (2004). Its main advantage is that it is stationary, and hence can be used directly. We have also considered linearly detrended per capita employment as a proxy for real activity with the same results. Chernov and Mueller (2008) report similar findings based on a quarterly analysis of the CPI and the linearly detrended GDP.

${ }^{10} \mathrm{We}$ are grateful to Robert Bliss for providing us with the data.
} 
is important to measure the full yield curve because its slope is correlated with the macro environment (Estrella and Hardouvelis, 1991; Estrella and Mishkin, 1998). Moreover, using rich yield data aids the identification of the risk premia.

One concern that arises in any study of interest rates over a relatively long period is that of structural stability. This issue is related to the monetary experiment of 1979-82 and the accompanying disinflation period. A common strategy is simply to consider the pre- and post-experiment samples separately, as in the work of Duffee (2006) and others. However, because of the persistence of interest rates, it is more desirable to study long data spans. $\mathrm{AP}$ and ADP, in settings similar to ours, find that subsamples and the full data set yield similar results. ${ }^{11}$ Perhaps the most appealing approach is to consider a regime-switching model, one that accounts explicitly for structural changes that are due to either the conduct of monetary policy or natural business cycles (e.g. Ang et al., 2004, or Dai et al., 2003). We proceed with our analysis bearing these caveats in mind.

\subsection{The Econometric Method}

We estimate our term structure model via maximum likelihood with the Kalman filter, following the work of Duffee and Stanton (2004), de Jong (2000), and others. ${ }^{12}$ We place estimation errors on all yields so that the latent factors are not associated with pre-specified maturities. We assume that the macro variables are observed without error. There are many compelling arguments in favour of introducing macro-measurement errors. The macro-measurement concerns are mitigated in our setting because we assume that investors react directly to the CPI and the HWI.

\footnotetext{
${ }^{11}$ We have performed some robustness checks for the pre-1979 and post-1984 subsamples. These checks indicate that results obtained from subsamples that exclude the period of the monetary experiment are qualitatively similar to those obtained from the full sample. We will comment on these results in later sections while making reference to specific findings.

${ }^{12}$ Other important strategies for estimation that have been applied to term structure models include, but are not limited to, the exact inversion likelihood of Chen and Scott (1993), the closed-form approximate likelihood of Ait-Sahalia and Kimmel (2009), the simulated maximum likelihood of Brandt and He (2002), and the Bayesian MCMC of Collin-Dufresne et al. (2009).
} 


\subsubsection{Observation Equations}

The state equation in the state-space system is the Gaussian $\operatorname{VAR}(1)$ described in (1). The observation equations can be represented in the following way:

$$
y_{t}=a+b_{m}^{Q} m_{t}+b_{x}^{Q} x_{t}+\chi_{t}
$$

where $y$ represents a vector of eight yields of maturities from one month to 10 years. The right-hand side of the equation is an expanded version of the no-arbitrage expression for the yield in (5). Measurement errors are denoted by $\chi$. We assume the simplest possible structure of the errors, i.e., that they are independent and normally distributed with zero mean and standard deviation $\sigma_{\chi}$ (for each individual element of the vector $\chi$ ). We need not specify a more flexible error structure because these variables are introduced in addition to the VAR shocks that we considered earlier.

After estimating a model and filtering the state variables, we can use the projection (16) to construct our interest rate decomposition (17). We will denote the filtered state variables by $\hat{x}\left(m^{t}, y^{t}\right)$, where, as before, superscripts denote the entire history of $m$ and $y$, respectively, up to time $t .{ }^{13}$ The orthogonalized residual is then estimated as $\hat{f}_{t}=\hat{x}\left(m^{t}, y^{t}\right)-\hat{x}\left(m^{t}\right)$.

\subsubsection{Number of Factors and Identification}

We estimate a model that has a total of four factors: $x_{t}=\left(x_{1 t}, x_{2 t}\right)^{\prime}$. Our principal component analysis (available upon request) suggests that at least four factors are required to explain the joint variation in the macro variables and yield data. We also estimated a three-factor model, but discovered that it could not capture the slope of the yield curve. ${ }^{14}$ For these reasons, we aim to identify a maximally flexible four-factor model with two observable factors.

\footnotetext{
${ }^{13}$ Note the difference between the filter of the latent variable $x$ that is based on all observables, $\hat{x}\left(m^{t}, y^{t}\right)$, and the projection of the latent variable $x$ onto the history of macro variables, $\hat{x}\left(m^{t}\right)$, discussed in the section 3.2.

${ }^{14}$ We will comment further on the three-factor model when we discuss empirical results.
} 
Dai and Singleton (2000) show that if all factors are latent in the Gaussian system, the parameters of the model can be identified provided $\mu=0, \Phi$ is lower triangular, $\Sigma$ is diagonal, and $\delta=1$. We also know from the literature on macro VARs that if all factors are observable, then $\mu, \Phi$, and $\delta$ are free, and $\Sigma$ is lower triangular. Given that we have a mixture of observed and latent factors, we have to combine the insights from the two strands of the literature.

We allow $\mu^{m}, \Phi, \delta_{0}$, and $\delta_{m}$ to vary freely. We restrict $\mu^{x}$ in such a way that the long-run mean of the factors $x$ is equal to zero, i.e.

$$
e_{i}^{\prime}(I-\Phi)^{-1} \mu=0
$$

where $e_{i}$ 's are vectors of zeros with a one in positions corresponding to the factors $x$ and $\Sigma$ is

$$
\Sigma=\left[\begin{array}{cccc}
\sigma_{g g} & 0 & 0 & 0 \\
\sigma_{\pi g} & \sigma_{\pi \pi} & 0 & 0 \\
\sigma_{1 g} & \sigma_{1 \pi} & \sigma_{11} & 0 \\
\sigma_{2 g} & \sigma_{2 \pi} & 0 & \sigma_{22}
\end{array}\right] .
$$

These restrictions entail that we have to set $\delta_{x}=1$. Finally, given that all the risk premia parameters are identified in the case of the all-latent model so long as there are more yields than factors, these parameters will be identified when some of the factors are observable.

A final piece of identification pertains to the indeterminacy of latent factors. While we have just imposed certain identifying restrictions, Dai and Singleton (2000) point out that such restrictions are not necessarily unique. There are many possible sets of restrictions, corresponding to transformations of the model that leave the yields unchanged. Naturally, when a parameter configuration changes, the respective latent variables change as well by "rotating." Given that the goal of this work is to disentangle the contributions of various 
factors, it is sensible to rotate the factors $f$ such that they are mutually orthogonal; i.e., so that $P$, the variance-covariance matrix of $f$, becomes diagonal (see the expression for $P$ in Appendix A). We use an invariant affine transformation, which scales factors by a matrix. Specifically, we rotate the factors $f$ into $R f_{t}$, where the matrix $R$ is such that $R P R^{\prime}$ is diagonal. Appendix A of Dai and Singleton (2000) describes how such a transformation affects model parameters.

\subsubsection{Estimation of the Risk Premia}

Risk premia are difficult to estimate in practice, despite their theoretical identification. Typically, one encounters multiple local optima that have similar likelihood values but that imply dramatically different estimates of the risk premia. In addition, adopting too rich a specification of the market prices of risk might be cause for concern. Such richness may compensate for misspecifying the factor dynamics instead of measuring the compensation for risk.

In order to mitigate the possible effect of these issues, we augment the standard log-likelihood function, $\mathcal{L}$, with a penalization term that is proportional to the variation of the term premium in (5):

$$
\mathcal{L}_{p}=\mathcal{L}-\frac{1}{2 \sigma_{p}^{2}} \sum_{\tau}\left(a^{T P}(\tau)\right)^{2}+b^{T P}(\tau)^{\prime} \cdot \operatorname{Diag}\left(\operatorname{var}\left(z_{t}\right)\right) \cdot b^{T P}(\tau)
$$

where $\sigma_{p}$ controls the importance of the penalization term and the Diag operator creates a diagonal matrix out of a regular one. If the market prices of risk are equal to zero, the term premium will be equal to zero as well. Therefore, $\mathcal{L}_{p}$ imposes an extra burden on the model to use the risk premia as a last resort when fitting the yields.

In practice, we take $\sigma_{p}=300$, thereby introducing a modest modification to the original log-likelihood function. This choice helps to stabilize the likelihood and simplifies the search for a global optimum. In particular, this setup helps us avoid any local maxima that are associated with very large risk premia. 


\section{The Contribution of Macro Factors to the Yield Curve}

The estimated model does a good job fitting the key moments of the data. Details of the estimated parameters and our diagnostics are provided in Appendix B. The key conclusion to be drawn from these diagnostics is that the model is sufficiently realistic for its implications to be taken seriously. In this section, we rely on the estimated model and our projection procedure to explore the issues that we raised in earlier sections.

\subsection{The sensitivity of the variance decomposition to the shock identification scheme}

Before we proceed with an empirical illustration of the projection approach, we show what kind of answers regarding the association between the macro variables and yields we can get using the standard VAR approach. Specifically, we compute the variance decomposition of the yield curve that corresponds to the infinite horizon forecast. As mentioned earlier, this is equivalent to unconditional variance of the yields. We summarize the yield curve by its first three principal components: level $y_{t}(3)$, slope $y_{t}(120)-y_{t}(3)$, and curvature $y_{t}(3)+y_{t}(120)-2 \cdot y_{t}(24)$. We have to choose the order of state variables to implement the recursive identification scheme. Given that we have no guidance from theory, we decided to consider all possible orders. Table 1 reports the joint contribution of shocks to real activity and inflation.

Typically, studies on macro-finance term structure order macro variables first, followed by latent variables. There are four possible combinations that correspond to this strategy: $\left(g, \pi, x_{1}, x_{2}\right),\left(g, \pi, x_{2}, x_{1}\right),\left(\pi, g, x_{1}, x_{2}\right)$, and $\left(\pi, g, x_{2}, x_{1}\right)$. The variance decompositions corresponding to these orderings are highlighted in italics in the Table. Within these four choices, the specific order does not matter. However, the contribution of macro shocks to the level computed based on these orderings, that is, $52 \%$ is right in the middle of the possible range from $36 \%$ for $\left(x_{2}, \pi, g, x_{1}\right)$ to $77 \%$ for $\left(x_{1}, g, \pi, x_{2}\right)$. Similarly, according to the variance decomposition, macro variables can explain anywhere from $25 \%$ to $81 \%$ of the variation in slope and from $32 \%$ to $88 \%$ of the variation in curvature. 
These numbers suggest that, in fact, the conclusions about the magnitude of association between the yields and macro variables are extremely sensitive to the identification of shocks. One has to either (i) use a structural model that identifies shocks uniquely, or (ii) rely on the observables, that is, levels of the macro variables as opposed to shocks, to try to gauge the relationship in question.

\subsection{How much yield-curve variance is associated with macro variables?}

\subsubsection{Short Interest Rate}

In this section, we explore how macro factors contribute to variations in the short interest rate. Table 2 reports theoretical $R^{2}$ values for the full model and a nested, macro-only specification. The macro component of the interest rate rule (17) explains the majority of the variation in the short rate: $R_{m}^{2}=80 \% .{ }^{15}$ This number is large and statistically significant: the 2.5 th percentile is equal to $44 \%$. Given our discussion in section 3.3, this result allows us to reject the restriction $R P$ and quantify the magnitude of covariation between the short rate and macro factors. Panel (a) of Figure 1 complements our observations about the strong relationship.

Our methodology also allows us to describe how macro variables affect the short rate. Panel (b) of Figure 1 illustrates the weights of the macro variables and their lags as components of the interest rate rule. The highest loading for real activity is at its contemporaneous value. The weights decline from there forward in an exponential fashion. The inflation loadings peak at the second lag and then decline in a manner similar to real activity. We note that the weights die out quite slowly, which implies a need for lags well beyond the traditional 12. Implementing such a lag structure in the VAR framework would be difficult because of

$\overline{{ }^{15} R_{m}^{2}}$ is equal to $72 \%$ in a three-factor version of our model with one latent variable. $R_{m}^{2}$ is equal to $76 \%$ and $77 \%$ for the pre-1979 and post-1984 subsamples, respectively, based on the same set of parameter values. These results stand in stark contrast to previous results published in the noarbitrage literature: AP report an $R^{2}=45 \%$ based on contemporaneous macro variables only and $R^{2}=53 \%$ for an interest rate rule that involves both macro variables and their lags and latent variables. Dai and Philippon (2004) report a high $R^{2}$ of $95 \%$ for their interest rate rule. However, this includes the contemporaneous Federal Funds rate. 
parameter proliferation.

The lag loadings illustrate that the implicit backward-looking interest rate rule is very different from the simple rule

$$
r_{t}=\delta_{0}+\delta_{m}^{\prime} m_{t}
$$

that is based on (2) and the assumption that latent factors $x$ represent monetary shocks (c.f. the benchmark Taylor rule in ADP). According to the estimated parameters $\delta_{m}$, reported in Table 4 , the short interest rate changes by $1.62 \%$ and $1.45 \%$ when the real activity and inflation change by $1 \%$, respectively. When we isolate those shocks that are orthogonal to the current macro variables and their histories, the picture becomes much more nuanced. In particular, the short interest rate changes by $0.1 \%$ and $0.01 \%$ in response to the $1 \%$ change in the contemporaneous real activity and inflation, respectively. ${ }^{16}$ Panel (a) of Figure 1 compares the simple and backward-looking rules. The simple rule is much more volatile and implies unrealistically large policy shocks. Such behavior on the part of the Federal Reserve Bank (the Fed), even if implicit, can hardly be imagined, given its goal of macroeconomic stabilization.

Finally, after fully incorporating the latent variables in (17), the $R_{z}^{2}$ is close to $100 \%$ in Table $2 .{ }^{17}$ This implies that the new latent factors $f$ explain the remaining $20 \%$ of the variation in the short rate.

\subsubsection{Slope and Curvature}

In this section, we establish what fraction of the variation in the yield curve is related to macro variables. As before, we summarize the yield curve by its first three principal

\footnotetext{
$\overline{16}$ These numbers are consistent with recent theoretical work in which it is argued that backwardlooking rules are more attractive. For example, the analysis in Benhabib et al. (2001) suggests that passive backward-looking policy rules stabilize the real side of economy, thus ensuring a unique equilibrium. In contrast, they claim that an aggressive, purely contemporaneous rule or a forwardlooking rule could give rise to multiple equilibria.

${ }^{17}$ Similarly, $R_{z}^{2}$ is close to $100 \%$ in a three-factor model.
} 
components: level $y_{t}(3)$, slope $y_{t}(120)-y_{t}(3)$, and curvature $y_{t}(3)+y_{t}(120)-2 \cdot y_{t}(24)$. As in the previous section, we consider two versions of the interest rate rule that are based on different implementations of our specifications in (17). In the first case, we omit the orthogonalized residuals $f$. In the second case, we use the full state vector.

Table 2 reports the theoretical $R^{2}$ values, which are based on the estimated model parameters. As we observed in the previous section, our model successfully explains the yield level using only macro variables. However, there is a large difference in the way in which the two interest rate rules explain the slope of the term structure. Our full model, which explains $97 \%$ of the variation, is certainly successful in capturing the slope of the curve. ${ }^{18}$ However, when only the real activity and inflation are considered, the ability of the model to capture the shape of the curve deteriorates. Although these two macro variables explain $80 \%$ of the variation in the level, they explain only $52 \%$ of the slope. ${ }^{19}$ By construction, this is the maximum amount of slope variation that can be explained by the two macro variables.

Figure 2(a) complements this discussion by contrasting the macro-based and observed slopes. Figure 2(b) shows the weights of the macro variables and their lags, which are used in the macro-based expression for the slope. In contrast to the short interest rate, the loadings on the current values of the macro variables are much larger than the loadings on their lags. Even so, the latter are non-zero and die out rather slowly, particularly in the case of real activity.

The power of the variables to explain the curvature follows roughly the same pattern, but our model fails to generate a realistic fit (the full model explains only $56 \%$ of the curvature variation). ${ }^{20}$ This outcome is consistent with the model diagnostics reported in Appendix B. While this problem could be remedied by introducing an extra latent variable, we decided to omit this refinement because the curvature contributes less than $1 \%$ to the variation in term structure.

$\overline{18}$ The three-factor model explains $48 \%$ of the slope.

${ }^{19}$ In the three-factor model, macro variables explain $39 \%$ of the slope.

${ }^{20}$ The three-factor model explains $17 \%$ of the curvature. 


\subsection{How much risk-premia variance is associated with macro-variables?}

The main conclusion so far is that macro variables covary in important ways not only with the short rate but with the rest of the yield curve. The issue remains of whether this covariation in the yield curve is related to the short rate or to the risk premia. Indeed, the yield equation (5) implies that the slope can be represented as

$$
\begin{aligned}
y_{t}(120)-y_{t}(3) \approx\left(a^{P}(120)-a^{P}(3)\right)+\left(b^{P}(120)-b^{P}(3)\right)^{\prime} z_{t}+a^{T P}(120)+b^{T P}(120)^{\prime} \neq(31) & \text { Risk premium exposure } \\
\text { Short rate exposure } & \text { Riste }
\end{aligned}
$$

because the three-month term premium is approximately zero. Thus, by focusing on the term premia, we can refine our analysis of the sources of variation in yields.

We can use our procedure to address this issue. Our analysis has direct implications for the role of risk premia, because the state variables that we have identified through our projectionbased decomposition affect the dynamics of the stochastic discount factor (3). Thus, we can decompose the term premia into macro variables and new latent variables in much the same way as we did with the yields. However, these state variables enter the risk premia with loadings different from the ones in the yields case. Therefore, we must test whether the macro contribution is significant.

\subsubsection{Decomposition of the Term Premia}

We break the term premia down into individual contributions of inflation and real activity using the projection-based representation. The yield equation (5) implies that the term premium of maturity $\tau$ can be expressed as

$$
\begin{aligned}
T P(\tau) & =a^{T P}(\tau)+b^{T P}(\tau)^{\prime} z_{t} \\
& =a^{T P}(\tau)+\underbrace{b_{g}^{T P}(\tau) g_{t}+\left.b_{x}^{T P}(\tau)^{\prime} \hat{x}\left(m^{t}\right)\right|_{g^{t}}}_{\equiv B_{g}(\tau) g^{t}}+\underbrace{b_{\pi}^{T P}(\tau) \pi_{t}+\left.b_{x}^{T P}(\tau)^{\prime} \hat{x}\left(m^{t}\right)\right|_{\pi^{t}}}_{\equiv B_{\pi}(\tau) \pi^{t}} \\
& +b_{x_{1}}^{T P}(\tau) f_{1 t}+b_{x_{2}}^{T P}(\tau) f_{2 t} .
\end{aligned}
$$


The term $\left.b_{x}^{T P}(\tau)^{\prime} \hat{x}\left(m^{t}\right)\right|_{g^{t}}$ denotes the part of the projection of the latent factors $x$, that depends on $g^{t}$, the current and lagged values of real activity only. We use similar notation for the inflation component. This breakdown allows us to characterize how the term premia vary together with changes in the macro variables and shocks.

Figure 3 displays the loadings $B_{g}(\tau)$ and $B_{\pi}(\tau)$ on the first 24 lags of the respective macro variables in the cases of one- and 10-year term premia. The 10 -year premium is more responsive to macroeconomic conditions than the one-year premium. The pattern of the two responses is otherwise qualitatively similar: term premia decline in response to a contemporaneous increase in either real activity or inflation. The loadings on the lagged values are positive, but smaller in magnitude. The loading on the first lag of both real activity and inflation is approximately 0.03 , which is comparable to the loading on inflation that is visible in the reaction function of Figure 1. Likewise, the weights gradually decline to zero as the lag order increases. Its contemporaneous loadings imply that the 10-year term premium increases by $0.4 \%(0.2 \%)$ in response to a $1 \%$ decline in real activity (inflation). This is consistent with our intuition that the term premia should be higher in recessions, when both real activity and inflation tend to be low.

Figure 4 shows time series for the one-year and 10-year term premia. The top panels plot the yields, the term premia, and the expectations for the short rate. We note that the term premia are generally countercyclical, which is consistent with the macro loadings that may be seen in Figure 3. The estimated premia also have reasonable magnitudes. For the one-year horizon, the average absolute premium is $0.73 \%$ with a standard deviation of $0.63 \%$. For the 10-year horizon, these numbers become $2.29 \%$ and $1.59 \%$, respectively.

The remaining panels show our breakdown of the term premium into the real activity, $B_{g}(\tau) g^{t}$, and inflation, $B_{\pi}(\tau) \pi^{t}$, components of the premia (intermediate row), and into the latent components of the premia, $b_{x_{1}}^{T P}(\tau) f_{1 t}$ and $b_{x_{2}}^{T P}(\tau) f_{2 t}$ (bottom row). We see that for the 10-year horizon, the real activity and inflation components of the premia are larger than the latent components. Furthermore, the real activity and inflation components tend to increase during recessions. The real activity component is less variable than the inflation 
component and smaller in magnitude, except for a period beginning in December 1998. At that time, the Fed started a tightening streak that led to the collapse of the stock market. Figure 4 indicates that concerns regarding the impact of these events on real activity dominated fears about inflation. The two latent components of the premia, on the other hand, often move in opposite directions.

\subsubsection{Variations in term premia}

We now compute the population variances of the macro components of term premia and evaluate their relative contribution to the variances of term premia. ${ }^{21}$ Due to the fact that the factors $f_{1}$ and $f_{2}$ are orthogonal to the macro factors, the decomposition of the term premia variance for a generic maturity $\tau$ simplifies to

$$
\operatorname{var}(T P(\tau))=\operatorname{var}\left(B_{g}(\tau) g^{t}+B_{\pi}(\tau) \pi^{t}\right)+\operatorname{var}\left(b_{x_{1}}^{T P}(\tau) f_{1 t}\right)+\operatorname{var}\left(b_{x_{2}}^{T P}(\tau) f_{2 t}\right)
$$

We compute the variance of the macro components by simulating a long path $(500,000$ observations) from the estimated model and computing the sample variance on its basis. We also compute the $95 \%$ confidence bounds for each factor's contribution to the variance, via the parametric bootstrap method described earlier.

Table 3 shows that our two macro variables (real activity and inflation) jointly contribute $54 \%$ to $68 \%$ of the variation in term premia. These numbers are significant. We have already rejected $R P$ on the basis of the $R^{2}$ for the short rate. Taken together with significant $R^{2}$ for the term premia, this result implies that $R Q$ are rejected. Therefore, the interest rate and risk premia are both important channels for joint covariation between the yields and the macro variables.

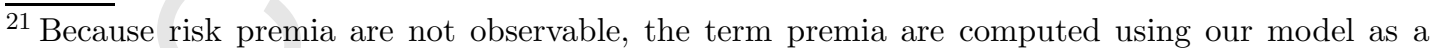
basis. Therefore, these relative contributions cannot be interpreted in the same way as the $R^{2}$ that we reported for the principal components. 


\subsection{Exogenous shocks or new systematic factors?}

In this section, we provide further, less formal, evidence regarding the nature of the latent factors $f$. First, we check whether we can interpret the latent factors as exogenous policy shocks, that is, as occasional responses of the Fed to economic indicators. Second, we would like to understand whether these factors are related to some observable macro variables, other than real activity and inflation, that could be affecting monetary policy in a systematic way.

\subsubsection{Exogenous shocks}

The deviations shown in Figure 1 between the interest rate rule and the short yield seem to support our desire to interpret the latent residual factors as exogenous shocks. Perhaps the most striking deviation from the macro-based rule occurs in the period from November 1982 to Spring 1986. Goodfriend (1993) associates this period with a particular episode: during the inflation scare of 1983 - 1984, the Fed established its credibility by aggressively increasing the funds rate by three percent. A similar, more recent episode pertains to the "soft landing" of 1994, when the Fed hiked interest rates by three quarters of a percentage point. Blinder and Yellen (2001) cite the years 1990 through 1993 as a period of passive policy. The Fed was accused of not cutting interest rates in a sufficiently proactive fashion during and after the recession of 1990-1991. This period was characterized by long pauses and small changes in interest rates. The deviations of the three-month yield from the macro-based rule shown in Figure 1 reflect this situation accurately.

As the interest rate rule of Figure 1 demonstrates, it is instructive to view the difference between the actual and macro-based slopes as a response of the yield curve to economic developments beyond those related to real activity and inflation. Indeed, factor $f_{1}$ accounts for almost the entire difference between the actual and macro-based slopes in Figure 2(a).

It is also interesting to contrast the plot of the slope with the two U.S. government budgetrelated episodes that are highlighted by Blinder and Yellen (2001). President Clinton introduced a budget reduction package in February 1993, immediately after the recession of 1990- 
1991. According to Blinder and Yellen, the unprecedented nature of this package awarded it instant credibility. This perception was mirrored by a $1.5 \%$ drop in the long interest rate over the year between late 1992, when Clinton started advertising the package, and late 1993. Given that the short interest rate was essentially flat at the time, this change in the actual slope may reasonably be attributed to changes in the fiscal policy. Another Clinton budget agreement, dated November 1999, “...shifted the norm for fiscal policy fundamentally by declaring the Social Security surplus off-budget..., the fiscal bar was thus raised enormously." Similar to the first episode, this announcement sparked a $2 \%$ drop in the slope between November 1999 and October 2000. Again, this can, to a large extent, be attributed to the change in fiscal policy, or in the context of our model to factor $f_{1}$.

The latent factor $f_{2}$ accounts for the entire difference between the interest rate rule and the short rate. It might seem surprising that $f_{1}$ does not affect the short rate. Indeed, our identification scheme imposes unit loading on $f_{1}$; this can be seen in the interest rate rule (17). In fact, the impact of either factor on the interest rate is controlled by its loading and by the variance of the factor's innovation. In our case, the estimated volatility of the innovation in $f_{1}$ is so small that it has a negligible effect on the short interest rate. The properties of the residual factors allow us to interpret them as an exogenous policy shock $\left(f_{2}\right)$ and an exogenous non-policy shock $\left(f_{1}\right)$.

\subsubsection{New Observable Factors}

The foregoing discussion suggests that the latent factors $f$ might be related systematically to macroeconomic factors other than inflation and real activity. The literature supports an interpretation of $f$ as a combination of monetary and fiscal shocks. The analysis of Mishkin and White (2003), for example, suggests that periods of financial instability are associated with liquidity crunches. Furthermore, these authors state that the Fed might react to important measures of financial instability, such as a large rise in interest rates for defaultable securities. Dai and Philippon (2004) argue that the budget deficit has an important effect on the entire yield curve. 
Our proxy for financial stability is the AAA credit spread, taken from the FRED database. Due to the overall high credit rating of AAA companies, the spread is a reasonable measure of the direct effects of liquidity. ${ }^{22}$ As an alternative measure of liquidity, we use the monthly growth in MZM as a direct measure of the money supply itself. We will also attempt to relate the shock $f_{1}$ to the yearly growth of the public government debt. We pre-whiten all three variables by regressing them on 12 lags of real activity and inflation. As a result, we relate the factors $f$ only to significant innovations in liquidity or fiscal policy that are unrelated to real activity and inflation. This is consistent with our interpretation of $f$ as the source of exogenous shocks.

Figure 5 plots factor $f_{1}$ against the pre-whitening residual of the annual growth of the public government debt. We find that the two series are quite strongly associated; the correlation between the two is $59 \%$. Figure 6 (a) plots the orthogonalized factor $f_{2}$ (with a minus sign) against the residual of the AAA spread. The two series share many spikes, which could be interpreted as "flights to quality" or more generically as liquidity events. The correlation between $f_{2}$ and the credit spread is $-38 \%$. This indicates that open market operations are occasionally related to liquidity; whether they react to it or cause it remains unknown. This is reflected in a separate market of quality corporate securities. An alternative measure of this effect can be obtained by correlating $f_{2}$ with the residual of the monetary aggregate MZM. Figure 6(b) shows the two variables. While their correlation is weak, at only $-12 \%$, a few periods of very strong association are still evident.

Is this evidence sufficient to conclude that the residual latent factors can be replaced by observable macro variables? Figures 5 and 6 show that there are periods during which the latent factors clearly have a strong association with our measures of liquidity and budget deficit. However, the relatively low $R^{2}$ values of the correlations indicate that there are even more periods when the interest rates are reacting to other sources of shocks. Thus, it appears more appropriate to model these shocks as latent factors rather than observable variables. It appears to be quite difficult to pinpoint one or two sources that consistently drive each

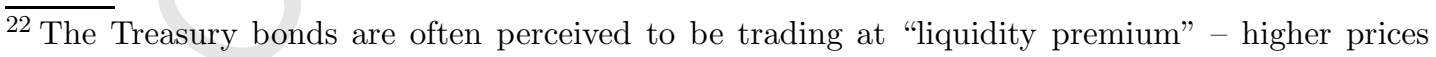
that reflect high demand for money-like instruments. 
factor. ${ }^{23}$

\section{Conclusion}

The macro-finance models of the yield curve combine the VAR dynamics of macro and latent factors with no-arbitrage restrictions to deliver implications about the joint behavior of the real and financial sectors. We show that using variance decomposition as a measure of the association between the macro and finance variables is not obvious, because its numerical values are sensitive to the order of variables used in the recursive identification scheme. We argue that the coefficient of determination, $R^{2}$, is a better measure because it relies on observable macro variables, as opposed to unobservable macro shocks. We propose a procedure that disentangles the contributions of macro and latent finance variables when they are correlated.

This methodology relies on two theoretical ingredients. First, we allow for a rich model specification that involves both macro variables (such as inflation and real activity) and latent factors. Second, we introduce new latent factors by dynamically projecting the traditional latent factors onto the macro variables. As a result, the linear relationship between the short interest rate and the model factors can be represented as a linear function of the macro variables, their lags, and the residuals of the orthogonal projection of latent factors.

We use the procedure to measure the contribution of the macro variables to the yield curve in the context of a four-factor model. We find that real activity, inflation, and their optimallyweighted lags explain $80 \%$ of the variation in the short interest rate. We find that the new latent variables (the projection residuals), which we interpret as exogenous monetary and fiscal shocks have a prominent impact on the short and long end of the yield curve, respectively. The shocks and the macro variables are equally important in explaining the

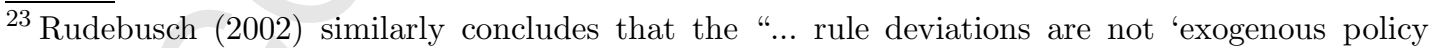
shocks,' that is, actions undertaken by central bankers that are independent of the economy ... Instead, these deviations are endogenous responses to a variety of influences that cannot be captured by some easily observable variable such as output or inflation." 
slope of the term structure.

We explore the impact of the macro variables on the term premia in our model. Inflation and real activity jointly explain $54 \%$ to $68 \%$ of the variation, depending on the horizon. We determine whether the residual latent variables, or shocks, are related to measures of liquidity (AAA spread, MZM growth) and the budget deficit (public debt growth). We find a fairly strong correlation between the former and monetary shock, and between the latter and the fiscal shock. Most strikingly, there are clear periods when the latent and the observable variables are associated with each other very strongly. However, there are prolonged periods when this relationship is weak. We conclude that our treatment of the shocks as latent variables is appropriate and that, most likely, they are correlated with different macro variables at different times. 


\section{References}

Aitt-Sahalia, Y., Kimmel, R., 2009. Estimating Affine multifactor term structure models using closed-form likelihood expansions, Journal of Financial Economics, forthcoming.

Ang, A., Bekaert, G., Wei, M., 2008. The term structure of real rates and expected inflation. Journal of Finance 63, 797-849.

Ang, A., Dong, S., Piazzesi, M., 2004. No-arbitrage Taylor rules, working paper, Columbia University.

Ang, A., Piazzesi, M., 2003. A no-arbitrage vector autoregression of term structure dynamics with macroeconomic and latent variables. Journal of Monetary Economics 50, 745-787.

Backus, D., Foresi, S., Telmer, C., 1999. Discrete time models of bond pricing. In: Jegadeesh, N., Tuckman, B. (Eds.), Advanced Fixed-Income Valuation Tools. Wiley and Sons, New York, Ny.

Bekaert, G., Cho, S., Moreno, A., 2010. New-Keynesian macroeconomics and the term structure. Journal of Money, Credit and Banking 42, 33-62.

Benhabib, J., Schmitt-Grohe, S., Uribe, M., 2001. Monetray policy and multiple equilibria. American Economic Review 91, 167-186.

Bils, M., Klenow, P. J., 2004. Some evidence on the importance of sticky prices. Journal of Political Economy 112, 947 - 985.

Blinder, A. S., Yellen, J. L., 2001. The Fabulous Decade. Macroeconomic Lessons from the 1990s. The Century Foundation Press, New York, NY.

Boivin, J., Bernanke, B., 2003. Monetary policy in a data-rich environment. Journal of Monetary Economics 50, 525-546.

Brandt, M. W., He, P., 2002. Simulated likelihood estimation of affine term structure models from panel data, working paper, Wharton.

Chen, R.-R., Scott, L. O., 1993. Maximum likelihood estimation of a multi-factor equilibrium model of the term structure of interest rates. Journal of Fixed Income , 14-31.

Chernov, M., Mueller, P., 2008. The term structure of inlation forecasts, CEPR Working paper No. 6809.

Christiano, L. J., Eichenbaum, M., Evans, C. L., 1999. Monetary policy shocks: What have 
we learned and to what end? In: Taylor, J., Woodford, M. (Eds.), Handobook of Macroeconomics. Elsevier Science, Amsterdam.

Collin-Dufresne, P., Goldstein, R., Jones, C., 2009. Can interest rate volatility be extracted from the cross section of bond yields? An investigation of unspanned stochastic volatility. Journal of Financial Economics 94, 47-66.

Conley, T., Hansen, L. P., Liu, W., 1997. Bootstrapping the long run. Macroeconomic Dynamics 1, 279-311.

Dai, Q., Philippon, T., December 2004. Fiscal policy and the term structure of interest rates, working paper, New York University.

Dai, Q., Singleton, K., 2000. Specification analysis of affine term structure models. Journal of Finance 55, 1943-1978.

Dai, Q., Singleton, K., 2002. Expectation puzzles, time-varying risk premia, and dynamic models of the term structure. Journal of Financial Economics 63, 415-441.

Dai, Q., Singleton, K., Yang, W., 2007. Regime shifts in a dynamic term structure model of U.S. Treasury bond yields. Review of Financial Studies 20, 1669-1706.

de Jong, F., 2000. Time series and cross-section information in affine term-structure models. Journal of Business and Economic Statistics 18, 300-314.

Diebold, F. X., Rudebusch, G. D., Aruoba, S. B., 2006. The macroeconomy and the yield curve: A dynamic latent factor approach. Journal of Econometrics 131, 309-338.

Duffee, G. R., 2006. Term structure estimation without using latent factors. Journal of Financial Economics 79, 507-536.

Duffee, G. R., 2007. Are variations in term premia related to the macroeconomy?, working paper, UC Berkeley.

Duffee, G. R., Stanton, R., 2004. Estimation of dynamic term structure models, working paper, University of California at Berkeley.

Estrella, A., Hardouvelis, G. A., 1991. The term structure as a predictor of real economic activity. Journal of Finance 46, 555-576.

Estrella, A., Mishkin, F., 1998. Predicting U.S. recessions: Financial variables as leading indicators. Review of Economics and Statistics 80, 45-61.

Evans, C. L., Marshall, D. A., September 2002. Economic determinants of the nominal trea- 
sury yield curve, working paper \#WP 2001-16 Revised, Federal Reserve Bank of Chicago.

Gallmeyer, M., Hollifield, B., Zin, S., 2005. Taylor rules, McCallum rules, and the term structure of interest rates. Journal of Monetary Economics 52, 921-950.

Goodfriend, M., 1993. Interest rate policy and the inflation scare problem: 1979-1992. Federal Reserve Bank of Richmond Economic Quarterly 79, 1-23.

Hamilton, J. D., 1994. Time Series Analysis. Princeton University Press, Princeton, NJ.

Hördahl, P., Tristani, O., Vestin, D., 2006. A joint econometric model of macroeconomic and term structure dynamics. Journal of Econometrics 131, 405-444.

Law, P., 2004. Macro factors and the yield curve, ph.D. dissertation, Stanford University.

Liptser, R. S., 1997. Stochastic control, Lecture notes, Tel Aviv University.

Litterman, R., Scheinkman, J., 1991. Common factors affecting bond returns. Journal of Fixed Income 3, 34-61.

Ludvigson, S. C., Ng, S., 2009. Macro factors in bond risk premia. Review of Financial Studies 22, $5027-5067$.

Mishkin, F., White, E. N., 2003. Stock market bubbles: When does intervention work? Milken Institute Review .

Mueller, P., 2008. Credit spreads and real activity, working paper, London School of Economics.

Rudebusch, G., 2002. Term structure evidence on interest rate smoothing and monetray policy inertia. Journal of Monetary Economics 49, 1161-1187.

Rudebusch, G., Wu, T., March 2005. A macro-finance model of the term structure, monetray policy and the economy, working paper, Federal Reserve Bank of San Francisco.

Woodford, M., 2003. Interest and Prices, 1st Edition. Princeton University Press, Princeton, NJ. 


\section{A Projection}

In this appendix, we first provide our projection formulas, then use them to show how our model is related to traditional VAR analysis.

\section{A.1 Recursive formulas}

We represent the state variable $z$ in (1) in block form:

$$
z_{t}=\left[\begin{array}{l}
\mu^{m} \\
\mu^{x}
\end{array}\right]+\left[\begin{array}{cc}
\Phi^{m m} & \Phi^{m x} \\
\Phi^{x m} & \Phi^{x x}
\end{array}\right]\left[\begin{array}{l}
m_{t-1} \\
x_{t-1}
\end{array}\right]+\left[\begin{array}{c}
\Sigma^{m m} \Sigma^{m x} \\
\Sigma^{x m} \Sigma^{x x}
\end{array}\right]\left[\begin{array}{l}
\epsilon_{t}^{m} \\
\epsilon_{t}^{x}
\end{array}\right],
$$

Liptser (1997) derives the projection of one element of the VAR(1) on the other using the same ideas as in a standard Kalman filter. In particular, Liptser derives the following expressions for the conditional mean, $\hat{x}\left(m^{t}\right)$, (often referred to as the "forecast"), and the covariance matrix, $P_{t}$, of the forecast errors:

$$
\begin{aligned}
\hat{x}\left(m^{t}\right) & =\mu^{x}+\Phi^{x x} \hat{x}\left(m^{t-1}\right)+\Phi^{x m} m_{t-1} \\
& +\left(\Sigma^{x x} \Sigma^{m x \prime}+\Sigma^{x m} \Sigma^{m m \prime}+\Phi^{x x} P_{t-1} \Phi^{m x \prime}\right)\left(\Sigma^{m x} \Sigma^{m x \prime}+\Sigma^{m m} \Sigma^{m m \prime}+\Phi^{m x} P_{t-1} \Phi^{m x \prime}\right)^{-1} \\
& \times\left(m_{t}-\mu^{m}-\Phi^{m x} \hat{x}\left(m^{t-1}\right)-\Phi^{m m} m_{t-1}\right) \\
P_{t} & =\Phi^{x x} P_{t-1} \Phi^{x x \prime}+\left(\Sigma^{x x} \Sigma^{x x \prime}+\Sigma^{x m} \Sigma^{x m \prime}\right) \\
& -\left(\Sigma^{x x} \Sigma^{m x \prime}+\Sigma^{x m} \Sigma^{m m \prime}+\Phi^{x x} P_{t-1} \Phi^{m x \prime}\right)\left(\Sigma^{m x} \Sigma^{m x \prime}+\Sigma^{m m} \Sigma^{m m \prime}+\Phi^{m x} P_{t-1} \Phi^{m x \prime}\right)^{-1} \\
& \times\left(\Sigma^{x x} \Sigma^{m x \prime}+\Sigma^{x m} \Sigma^{m m \prime}+\Phi^{x x} P_{t-1} \Phi^{m x \prime}\right)^{\prime} .
\end{aligned}
$$

We introduce additional notation to describe the projection initialization. The long run mean and variance of $z$ are 


$$
\begin{aligned}
(I-\Phi)^{-1} \mu & =\left[\begin{array}{c}
\Theta^{m} \\
\Theta^{x}
\end{array}\right] \\
V & =\left[\begin{array}{cc}
V^{m m} & V^{m x} \\
V^{x m} & V^{x x}
\end{array}\right], \text { where } V \text { solves } V=\Phi V \Phi^{\prime}+\Sigma \Sigma^{\prime}
\end{aligned}
$$

The steady-state matrix $P$ satisfies

$$
\begin{aligned}
P & =\Phi^{x x} P \Phi^{x x \prime}+\left(\Sigma^{x x} \Sigma^{x x \prime}+\Sigma^{x m} \Sigma^{x m \prime}\right) \\
& -\left(\Sigma^{x x} \Sigma^{m x \prime}+\Sigma^{x m} \Sigma^{m m \prime}+\Phi^{x x} P \Phi^{m x \prime}\right)\left(\Sigma^{m x} \Sigma^{m x \prime}+\Sigma^{m m} \Sigma^{m m \prime}+\Phi^{m x} P \Phi^{m x \prime}\right)^{-1} \\
& \times\left(\Sigma^{x x} \Sigma^{m x \prime}+\Sigma^{x m} \Sigma^{m m \prime}+\Phi^{x x} P \Phi^{m x \prime}\right)^{\prime}
\end{aligned}
$$

The projection is then initialized as follows:

$$
\hat{x}\left(m_{0}\right)=\Theta^{x}+V^{x m}\left(V^{m m}\right)^{-1}\left(m_{0}-\Theta^{m}\right), P_{0}=P
$$

In this case, $P_{t}=P$ and the projection is stationary. An alternative strategy is to initialize $P_{0}$ at the unconditional variance of $z$. In this case, the sequence $P_{t}$ will eventually converge to $P$. In our model, this convergence is quite rapid: it requires only 12 iterations.

\section{A.2 Relation to VAR}

Recall from Equation (15) that

$$
f_{t}=x_{t}-\hat{x}\left(m^{t}\right) .
$$

By construction, its variance is equal to $P_{t}$. Equations (A.1) and (A.2) imply that 


$$
\begin{aligned}
f_{t} & =\Phi^{x x} f_{t-1}+\Sigma^{x x} \epsilon_{t}^{x}+\Sigma_{x m} \epsilon_{t}^{m} \\
& -\underbrace{\left(\Sigma^{x x} \Sigma^{m x \prime}+\Sigma^{x m} \Sigma^{m m \prime}+\Phi^{x x} P_{t-1} \Phi^{m x \prime}\right)\left(\Sigma^{m x} \Sigma^{m x \prime}+\Sigma^{m m} \Sigma^{m m \prime}+\Phi^{m x} P_{t-1} \Phi^{m x \prime}\right)^{-1}}_{\mathcal{P}_{t-1}} \\
& \times\left(\Phi^{m x} f_{t-1}+\Sigma^{m x} \epsilon_{t}^{x}+\Sigma^{m m} \epsilon_{t}^{m}\right) \\
& =\left(\Phi^{x x}-\mathcal{P}_{t-1} \Phi^{m x}\right) f_{t-1}+\left(\Sigma^{x x}-\mathcal{P}_{t-1} \Sigma^{m x}\right) \epsilon_{t}^{x}+\left(\Sigma^{x m}-\mathcal{P}_{t-1} \Sigma^{m m}\right) \epsilon_{t}^{m}
\end{aligned}
$$

Therefore, the "residual" factors $f$ follow a VAR(1) process. However, in contrast with the dynamics of $m$ and $x$, the conditional mean and variance of $f$ are state-dependent. The steady-state Kalman filter theory implies that $\mathcal{P}_{t}$ converges to a fixed matrix together with $P_{t}$.

Let $\mathcal{P}=\lim _{t \rightarrow \infty} \mathcal{P}_{t}$. We introduce the following explicit notation for simplified referencing: ${ }^{24}$

$$
\begin{aligned}
\Phi^{f f} & \equiv \Phi^{x x}-\mathcal{P} \Phi^{m x} \\
\Sigma^{f f} & \equiv \Sigma^{x x}-\mathcal{P} \Sigma^{m x} \\
\Sigma^{f m} & \equiv \Sigma^{x m}-\mathcal{P} \Sigma^{m m}
\end{aligned}
$$

This yields

$$
\left[\begin{array}{c}
m_{t} \\
f_{t}
\end{array}\right]=\left[\begin{array}{l}
\mu^{m} \\
0
\end{array}\right]+\left[\begin{array}{cc}
\Phi^{m m} & \Phi^{m x} \\
0 & \Phi^{f f}
\end{array}\right]\left[\begin{array}{l}
m_{t-1} \\
f_{t-1}
\end{array}\right]+\left[\begin{array}{c}
\Phi^{m x} \hat{x}\left(m^{t-1}\right) \\
0
\end{array}\right]+\left[\begin{array}{c}
\Sigma^{m m} \Sigma^{m x} \\
\Sigma^{f m} \Sigma^{f f}
\end{array}\right]\left[\begin{array}{c}
\epsilon_{t}^{m} \\
\epsilon_{t}^{x}
\end{array}\right]
$$

Recursive substitution of $\hat{x}$ from (A.2) and the bond valuation of Equation (2) into (A.13) yields the VAR system (25).

\footnotetext{
$\overline{24}$ The identification assumptions regarding the matrix $\Sigma(28)$ simplify many projection equations.
} One notable simplification is that $\Sigma^{f f}=\Sigma^{x x}$. 


\section{B Estimation Results}

\section{B.1 Parameter Estimates}

Table 4 presents the estimated parameters. Because asymptotic standard errors are a concern in the context of persistent time series such as interest rates, we compute confidence bounds via the parametric bootstrap (Conley et al., 1997). Specifically, we simulate 1000 paths from the estimated model and re-estimate it along each path. This procedure yields a finite sample distribution of parameter estimates, which is subsequently used to determine the confidence intervals.

Although some parameters are individually insignificant, they appear to be jointly significant when our parameter elimination routine is applied. Specifically, following Dai and Singleton (2002), we restricted some of the insignificant parameters to zero, provided that this restriction did not lead to a notable decline in the value of the log-likelihood function. The remaining parameters are important for the model fit. The insignificance of individual parameters stems from the fact that we are estimating a large model; the data might not be sufficiently informative about each of the parameters, even if they are identified theoretically. ${ }^{25}$

Christiano et al. (1999) caution that the estimated parameters are difficult to interpret because they represent the convolution of the parameters of the actual interest rate rule and of the parameters that represent the projection of the missing data onto the econometrician's dataset. Moreover, the values of parameters that are associated with the latent variables depend on the particular identification scheme. For example, the magnitude of the lowerright block of $\Sigma$ depends on the restricted value of $\delta_{x}$.

$\overline{25}$ Still, the insignificance of the factor loadings $\delta_{0}$ and $\delta_{m}$ is somewhat disturbing. We surmise that this effect is the manifestation of general difficulties in estimating the means of persistent variables. This problem is subdued in latent factor models because $\delta_{0}$ is typically fixed at the sample mean and the rest of $\delta$ 's are restricted to the value one. Because two of our factors are observable, we have to estimate the factors' means, and the respective loadings. 


\section{B.2 The Model Fit}

First, we present the value of $\sigma_{u}$ (the standard deviation of the error in the yield observation equation (26)), which is equal to 0.16 . This implies that the model values the bonds within 32 basis points $\left(2 \sigma_{u}\right)$. Specifically, as indicated in panel (a) of Table 5 , the average absolute pricing error ranges from 6.2 basis points for the one-year yield to 33 basis points for the 10-year yield. ${ }^{26}$ In this regard, the results are consistent with other macroeconomic studies (see ADP for a discussion).

We conduct a more thorough evaluation of the model performance by checking how well it fits certain moments. Again, we use the parametric bootstrap strategy and compute a finite sample distribution for each of the model-implied moments. ${ }^{27}$ Panel (b) of Table 5 reports the results. ${ }^{28}$

We see that the model successfully captures many important aspects of the data, such as means, standard deviations, kurtosis, and autocorrelations. The difference between the fitted and actual means of the yields is quite large, at least relative to the existing studies. There are two reasons for this discrepancy. First, we do not assume that the yields are observed precisely. Second, we estimate the coefficient $\delta_{0}$ in the interest rate rule: most studies fix it to exactly match the unconditional mean of the short yield.

Where our model runs into difficulties is with explaining the skewness of many of the variables and the curvature of the yield curve. The former is not surprising, because Gaussian models are incapable of generating skewness. As for the curvature, the differences between the data and the model moments are significant. We intentionally selected a parsimonious model in this paper to focus on first-order effects and to minimize the already formidable difficulties

\footnotetext{
$\overline{26}$ The magnitudes of the pricing errors is largely similar in the post-1984 subsample given the same parameter values. However, the errors in the pre-1979 subsample are slightly higher, by two basis points on average, than for the full sample.

${ }^{27}$ In principle, we should have taken the parameter uncertainty and data sampling error into account when computing the confidence intervals. This would widen the intervals further.

${ }^{28}$ We have also tested how well the model fits the pre-1979 and post-1984 subsamples with the same set of parameter values. The results (available upon request) are largely unchanged.
} 
associated with a large set of parameters. The curvature fit can be improved by adding another latent factor. We do not pursue such extensions here, because the curvature does not have a first-order effect: it explains at most one percent of the variation in the yield curve.

\section{B.3 The Orthogonalized Residuals, $f_{t}$}

Table 6 reports the correlations between the filtered latent variables $x, \hat{x}\left(m^{t}, y^{t}\right)$ and the estimated orthogonalized residuals $\hat{f}_{t}$. We see that both $f_{\mathrm{s}}$ are quite different from their $x$ counterparts (the correlations are 0.22 and 0.31 for the first and second pairs, respectively). These low correlations imply that inferring the impact of macro variables with $x$ as the latent factors is very different from using $f$. Moreover, the correlations between the traditional latent factors (i.e., level, slope, and curvature) and either $x \mathrm{~s}$ or $f_{\mathrm{s}}$ are not as strong as in the latent factor models. The strongest relationship is between the slope and $x_{1}$ or $f_{1}$ (the correlation is above 0.60 in both cases). Finally, $f_{1}$ and $f_{2}$ are persistent with the respective serial correlations of 0.92 and 0.90 , but less so that the yields or macro variables (compare with panel (b) of Table 5).

These results indicate that our orthogonalization procedure was worth pursuing, because it leads to new latent variables that are substantially different from those typically studied in the literature. Real activity and inflation variables have a greater potential to explain the yield curve than they appear to if we consider the interest rate rule (2) directly. 
Table 1

Variance decompositions

We list the contribution of macro shocks to the variance in infinite horizon forecasts, or, equivalently, the unconditional variance, of the yield curve. We represent the yield curve by its first three principal components: level, $y(3)$, slope, $y(120)-y(3)$, and curvature, $y(3)+y(120)-2 \cdot y(24)$. We use the recursive shock identification scheme and provide variance decompositions for all possible orders of the state variables. We denote the variables $g, \pi, x_{1}$, and $x_{2}$ by $1,2,3$, and, 4 , respectively. We report the joint contribution of shocks to $g$ and $\pi$. The numbers are sorted according to the contribution of the shocks to the level of the yield curve. The italicized numbers correspond to the order of the state variables that is used in all studies.

\begin{tabular}{cccc}
\hline order & level & slope & curv \\
\hline 4213 & 36.47 & 24.88 & 37.88 \\
3241 & 38.98 & 44.71 & 50.40 \\
2413 & 41.76 & 61.84 & 41.06 \\
2431 & 43.29 & 26.30 & 45.14 \\
4231 & 43.80 & 51.63 & 32.16 \\
2314 & 44.30 & 51.15 & 50.91 \\
4312 & 49.31 & 38.37 & 54.26 \\
3412 & 49.31 & 38.37 & 54.26 \\
4321 & 49.31 & 38.37 & 54.26 \\
3421 & 49.31 & 38.37 & 54.26 \\
1234 & 51.64 & 36.90 & 41.72 \\
2143 & 51.64 & 36.90 & 41.72 \\
2134 & 51.64 & 36.90 & 41.72 \\
1243 & 51.64 & 36.90 & 41.72 \\
2341 & 53.10 & 50.80 & 63.21 \\
3214 & 53.65 & 45.60 & 47.58 \\
3142 & 56.58 & 62.60 & 63.63 \\
1423 & 58.69 & 52.51 & 48.44 \\
4123 & 59.68 & 60.17 & 68.26 \\
1324 & 61.23 & 41.81 & 58.29 \\
4132 & 61.40 & 69.51 & 45.40 \\
1432 & 65.83 & 34.37 & 69.66 \\
1342 & 75.64 & 58.87 & 87.74 \\
3124 & 76.86 & 80.90 & 77.95 \\
\hline & & &
\end{tabular}


Table 2

Theoretical $R^{2}$ for Principal Components

We establish which fraction of the yield curve variation is explained by various versions of the interest rate rule. We represent the yield curve by its first three principal components: level, $y(3)$, slope, $y(120)-y(3)$, and curvature, $y(3)+y(120)-2 \cdot y(24)$. We consider two versions of the interest rule, which depend on the difference in how the factors are used and on how the rules are estimated. The different rules are derived from the no-arbitrage model. Given the estimated coefficients, we compute the cumulative variance decomposition, i.e., the fraction of the unconditional variance that is explained. First, we evaluate the rule using the lagged macro variables

$$
r_{t}=\delta_{0}+\delta_{m}^{\prime} m_{t}+\delta_{x}^{\prime} \hat{x}\left(m^{t}\right)
$$

Next we use the full set of state variables:

$$
r_{t}=\delta_{0}+\delta_{m}^{\prime} m_{t}+\delta_{x}^{\prime} \hat{x}\left(m^{t}\right)+\delta_{x}^{\prime} f_{t} \equiv \delta_{0}+\delta_{m}^{\prime} m_{t}+\delta_{x}^{\prime} x_{t}
$$

The bootstrapped $95 \%$ confidence intervals are reported in parentheses.

\begin{tabular}{lrr}
\hline $\mathrm{PC}$ & $m_{t}+$ lags & full model \\
\hline level & 79.59 & 99.75 \\
& $(43.86,90.21)$ & $(99.21,99.86)$ \\
slope & 52.36 & 97.45 \\
& $(22.36,75.77)$ & $(95.17,98.43)$ \\
curvature & 42.74 & 55.83 \\
& $(8.43,64.04)$ & $(24.87,72.70)$ \\
\hline
\end{tabular}


Table 3

Decomposition of the Unconditional Variance of the Term Premia

We report the percentage contribution of macro risk factors to the overall unconditional variation in the term premia. We consider three maturities: one, five, and 10 years. The bootstrapped $95 \%$ confidence intervals are reported in parentheses.

\begin{tabular}{rrrr}
\hline & $m_{t}+$ lags & $f_{1 t}$ & $f_{2 t}$ \\
\hline 1-year & 53.70 & 4.44 & 41.84 \\
& $(25.44,77.01)$ & $(0.00,15.76)$ & $(21.86,66.74)$ \\
5-year & 67.86 & 6.39 & 25.74 \\
& $(28.13,89.54)$ & $(0.00,34.26)$ & $(10.01,46.19)$ \\
10-year & 68.49 & 8.84 & 22.65 \\
& $(30.04,91.36)$ & $(0.00,44.34)$ & $(7.17,34.95)$ \\
\hline
\end{tabular}


Table 4

Estimated Parameters

The table lists the parameter values for our model:

$$
\begin{aligned}
z_{t} & =\left(g_{t}, \pi_{t}, x_{1 t}, x_{2 t}\right) \\
r_{t} & =\delta_{0}+\delta_{z}^{\prime} z_{t} \\
z_{t} & =\mu+\Phi z_{t-1}+\Sigma \epsilon_{t} \\
\Lambda_{t} & =\Lambda_{0}+\Lambda_{z} z_{t} \\
\log \xi_{t} & =-r_{t-1}-\frac{1}{2} \Lambda_{t-1}^{\prime} \Lambda_{t-1}-\Lambda_{t-1}^{\prime} \epsilon_{t}
\end{aligned}
$$

The values correspond to annualized yields and macro variables observed at the monthly frequency. The bootstrapped $95 \%$ confidence intervals are reported in parentheses. The parameters restricted by the identification requirements are highlighted by the letter combination 'id' in place of the confidence bounds. The dash '-' indicates that a parameter was restricted in the course of estimation. Note that we report the long-run mean, $(I-\Phi)^{-1} \mu$, of the state variables.

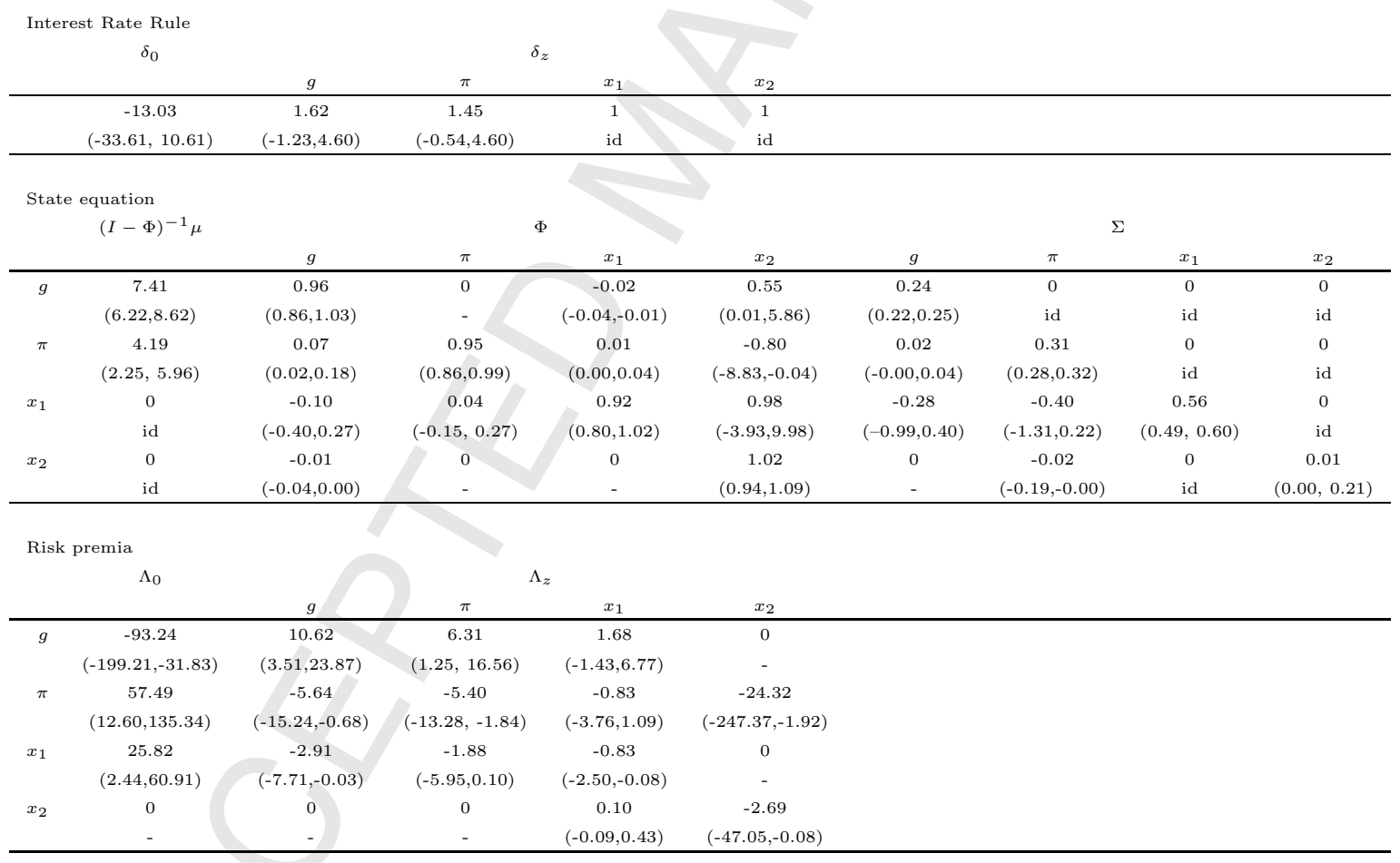


Table 5

Pricing Errors and Moments

We report average absolute pricing errors in yields by maturity in panel (a). Panel (b) reports various moments of the observables that were computed from the dataset (monthly observations from 1970 to 2002) and that are fitted by the estimated model. The bootstrapped $95 \%$ confidence intervals are reported in parentheses. The boldfaced sample statistics lie outside the confidence bounds.

Panel (a). Pricing Errors.

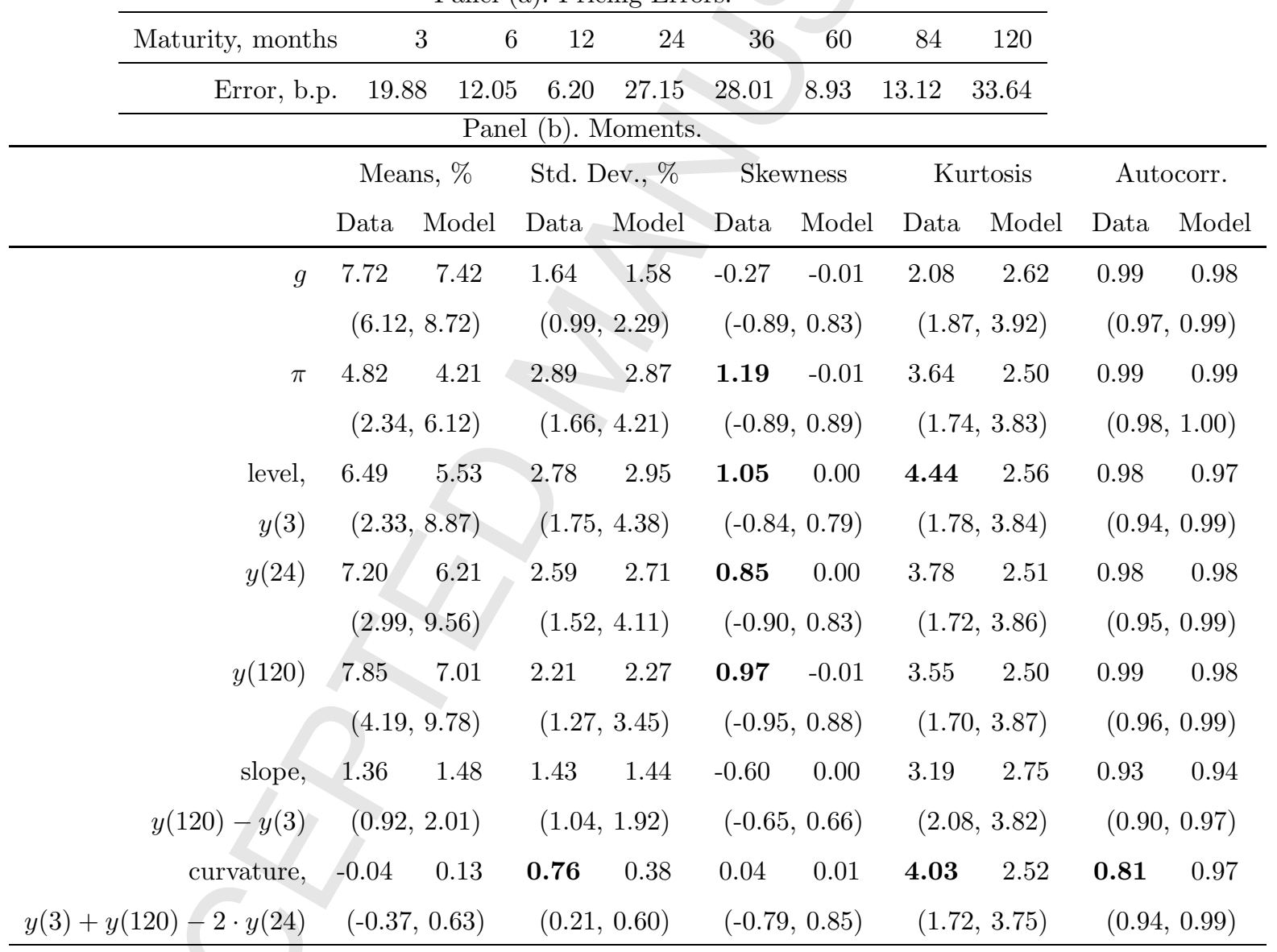


Table 6

Correlations Between the Traditional Latent Factors and the Residual Latent Factors

We correlate the three latent factors (level, $y(3)$, slope, $y(120)-y(3)$, and curvature, $y(3)+y(120)-2 \cdot y(24))$, which jointly capture $99 \%$ of the yield curve variation with two sets of latent factors that feature in our model. The factors $x$ enter our model directly, together with macro factors. The factors $f$ are residuals of projection of $x$ on to the history of the macro variables. We also report correlations between the respective $x \mathrm{~s}$ and $f \mathrm{~s}$. The low correlations imply that inferring the impact of macro variables with $x$ as latent factors is very different from using $f$.

\begin{tabular}{rrrrr}
\hline & $x_{1}$ & $x_{2}$ & $f_{1}$ & $f_{2}$ \\
\hline level & -0.38 & -0.26 & -0.04 & 0.60 \\
slope & 0.64 & 0.38 & 0.62 & -0.46 \\
curvature & -0.02 & 0.08 & -0.36 & -0.35 \\
\hline$x_{1}$ & & & 0.22 & \\
$x_{2}$ & & & & 0.31 \\
\hline
\end{tabular}


(a) Interest Rate Rule

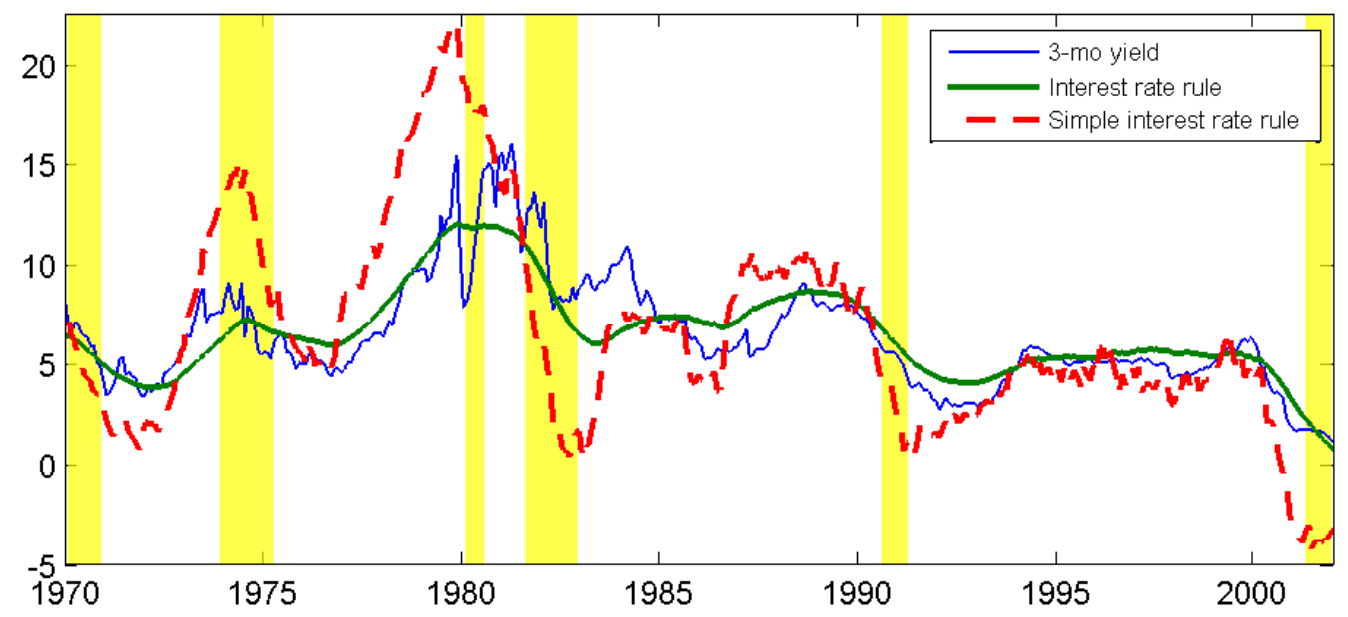

(b) Loadings on the macro variables in the interest rate rule

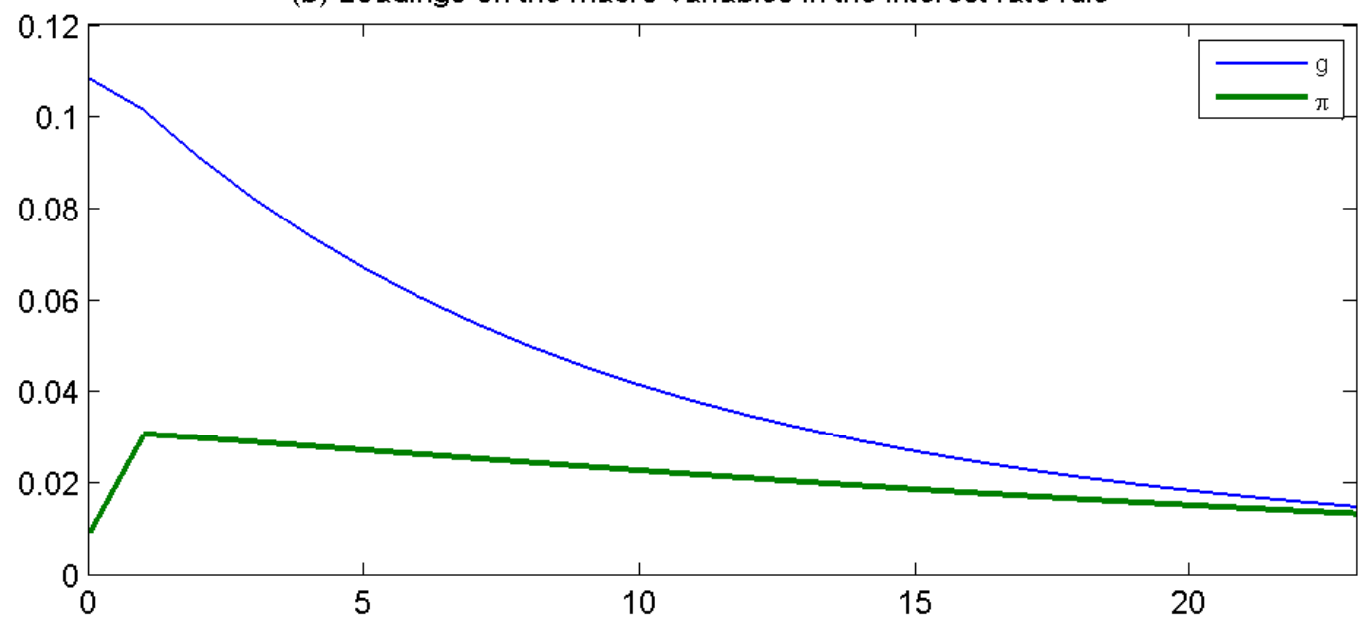

Fig. 1. We plot the time series of the three-month zero yield and the estimate of $r_{t}$ on the basis of the projection

$$
\hat{r}_{t}=\delta_{0}+\delta_{m}^{\prime} m_{t}+\delta_{x}^{\prime} \hat{x}\left(m^{t}\right)
$$

in panel (a). Panel (b) shows the first 24 loadings on the macro variables and their lags that generate the interest rate rule. 
(a) Slope implied by the interest rate rule

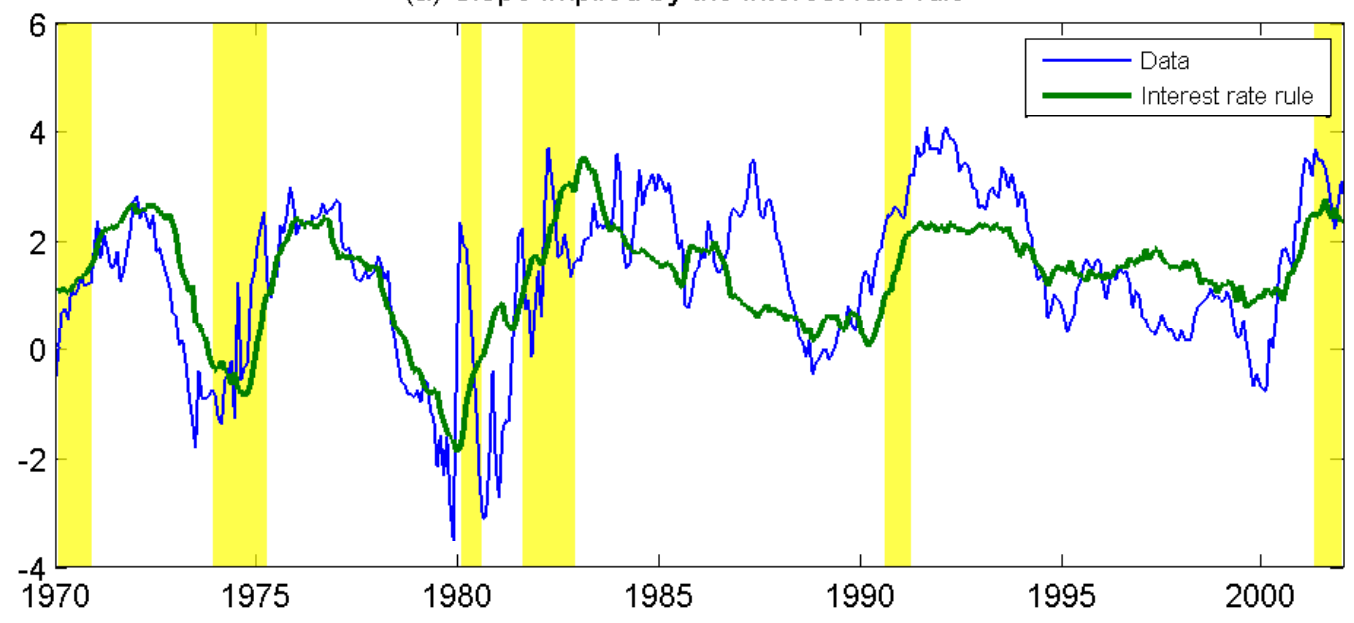

(b) Loadings on the macro variables in the interest rate rule

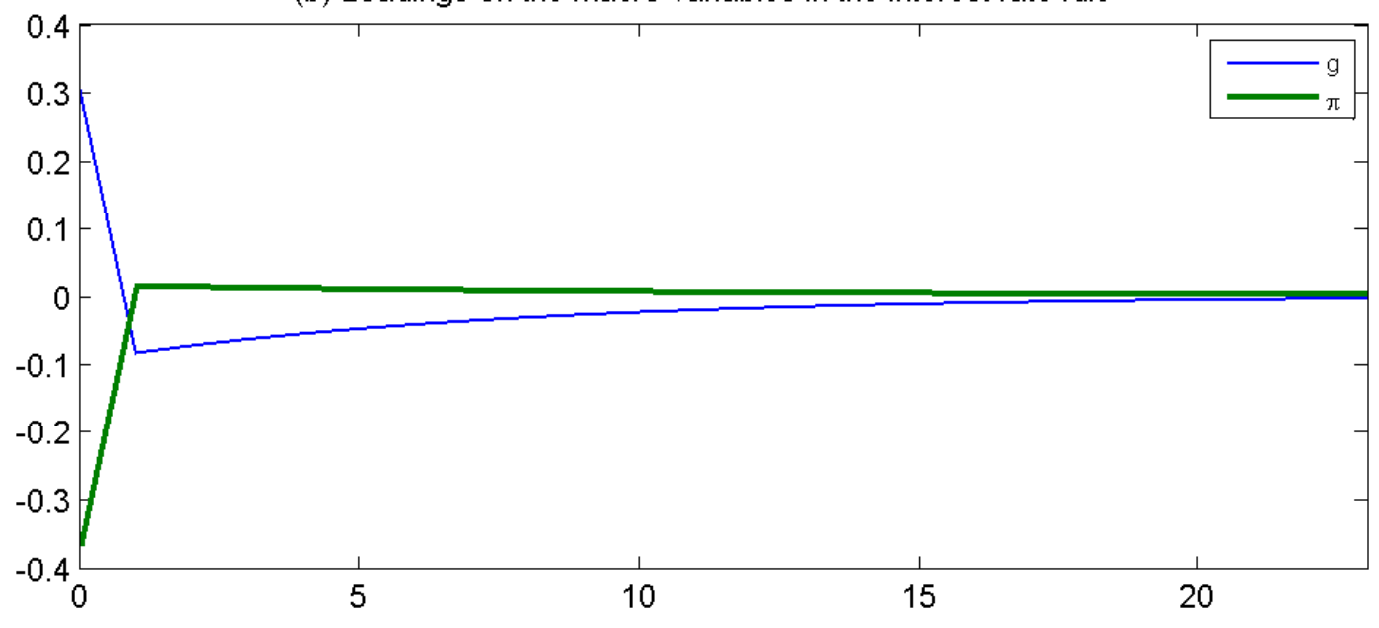

Fig. 2. We plot the time series of the slope and its estimate computed using the projectionbased interest rate rule

$$
\hat{r}_{t}=\delta_{0}+\delta_{m}^{\prime} m_{t}+\delta_{x}^{\prime} \hat{x}\left(m^{t}\right)
$$

in panel (a). Panel (b) shows the first 24 loadings on the macro variables and their lags, which generate the slope. 
(a) Loadings on the macro variables in the 1-year term premium

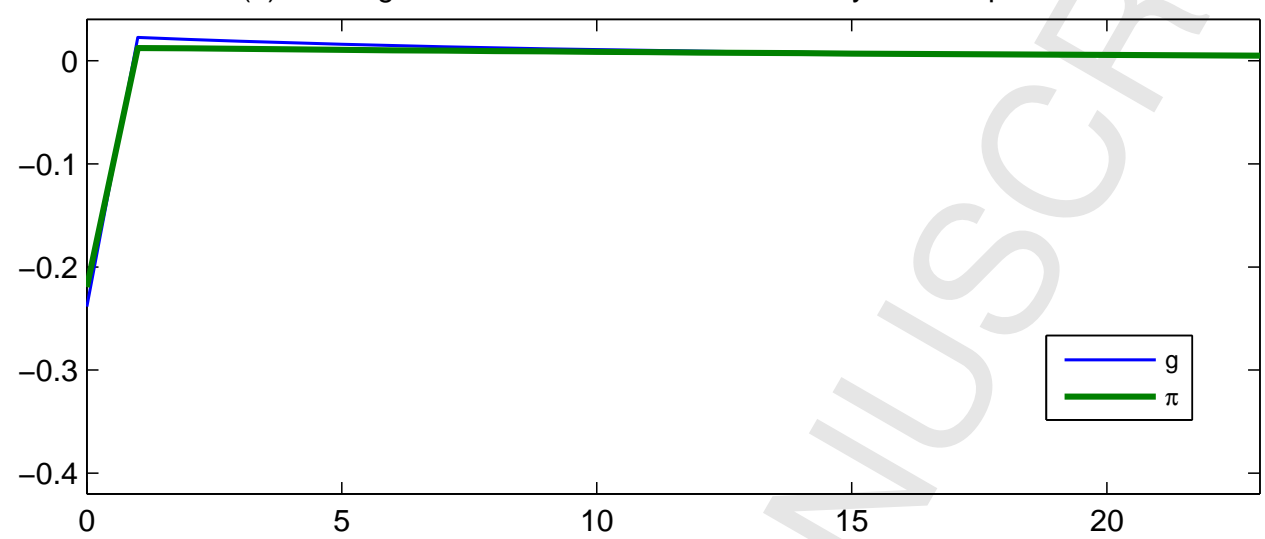

(b) Loadings on the macro variables in the 10-year term premium

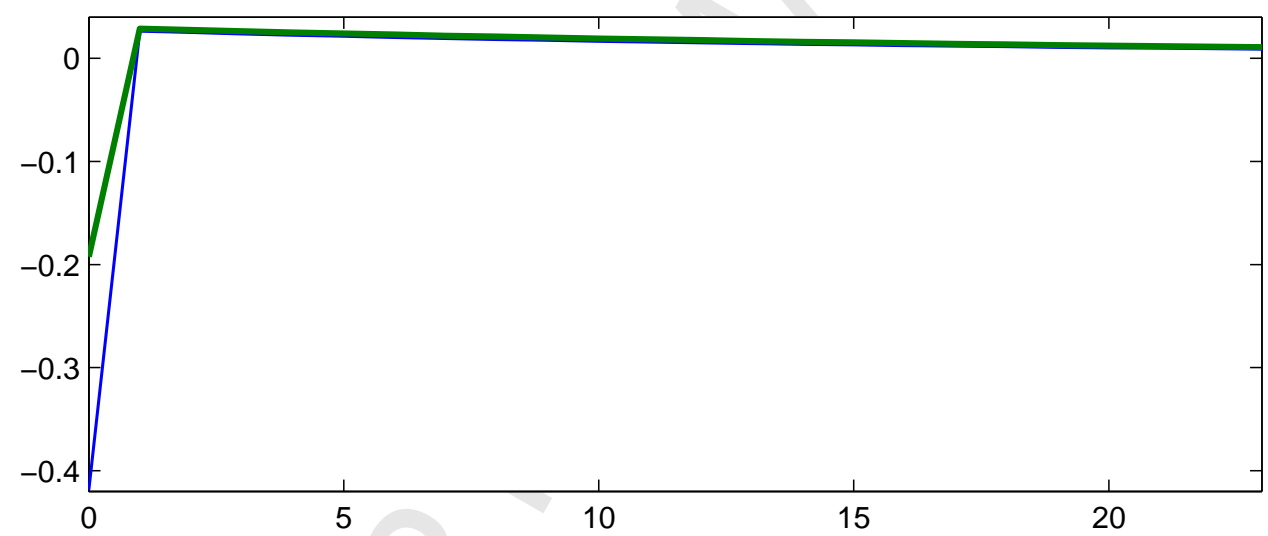

Fig. 3. We plot the first 24 loadings on the macro variables and their lags, which generate the term premia. 
Term Premium. 1 Year Maturity

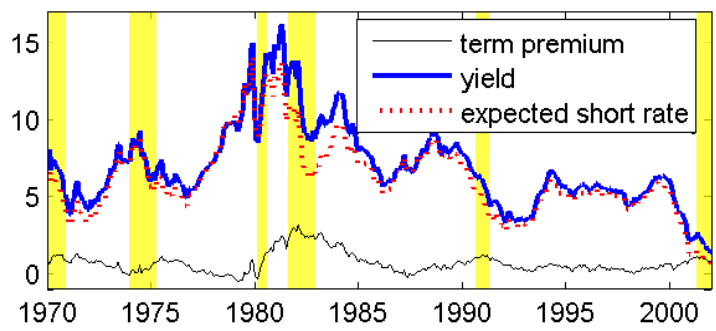

Term Premium Decomposition. 1 Year Maturity

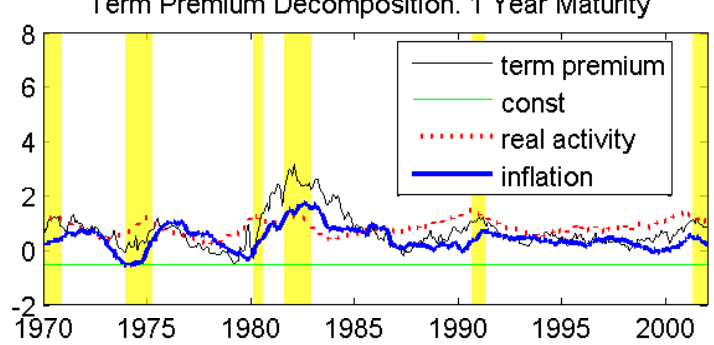

Term Premium Decomposition. 1 Year Maturity

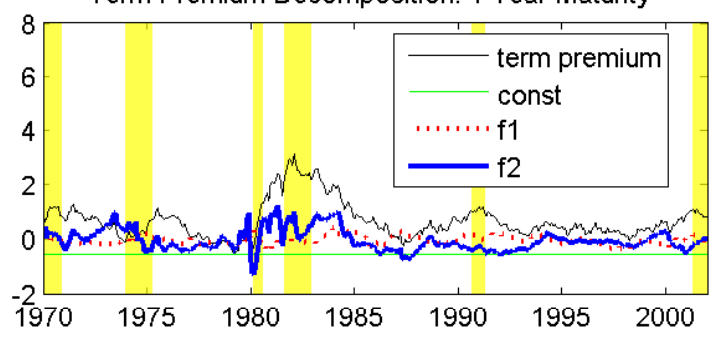

Term Premium. 10 Year Maturity

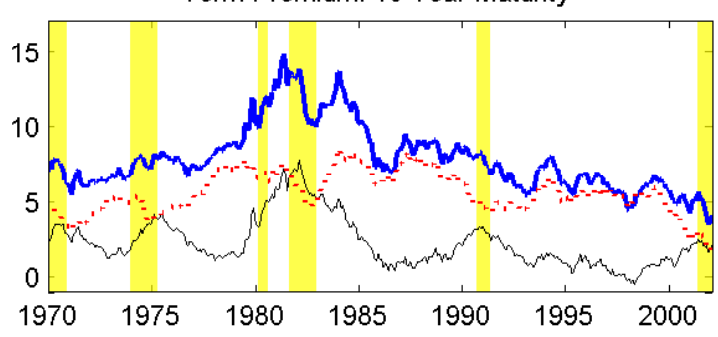

Term Premium Decomposition. 10 Year Maturity

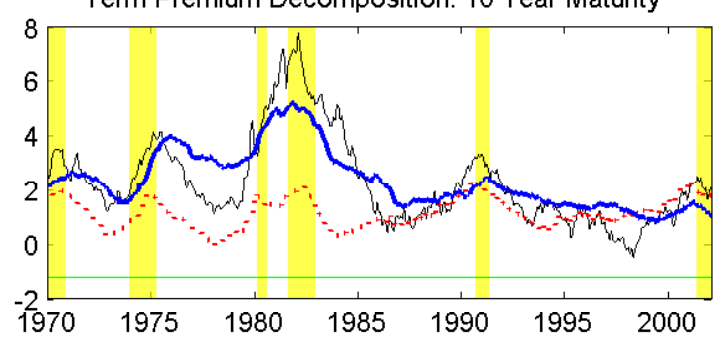

Term Premium Decomposition. 10 Year Maturity

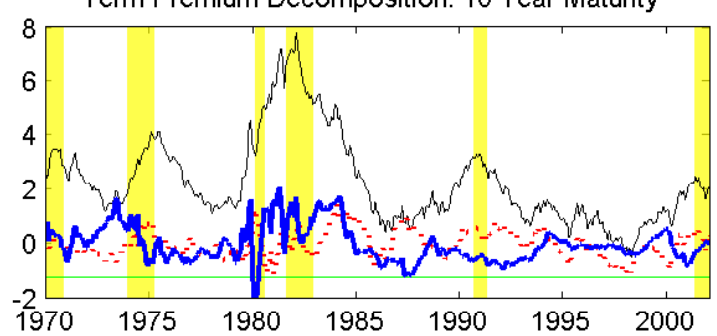

Fig. 4. The figure shows the time series of the one- and 10-year yields broken down into the expectations and term premia. The term premia are subsequently broken down into the contributions of the four determinants of the yield curve: real activity, inflation, fiscal and liquidity shocks. The shaded regions show the NBER recessions. 


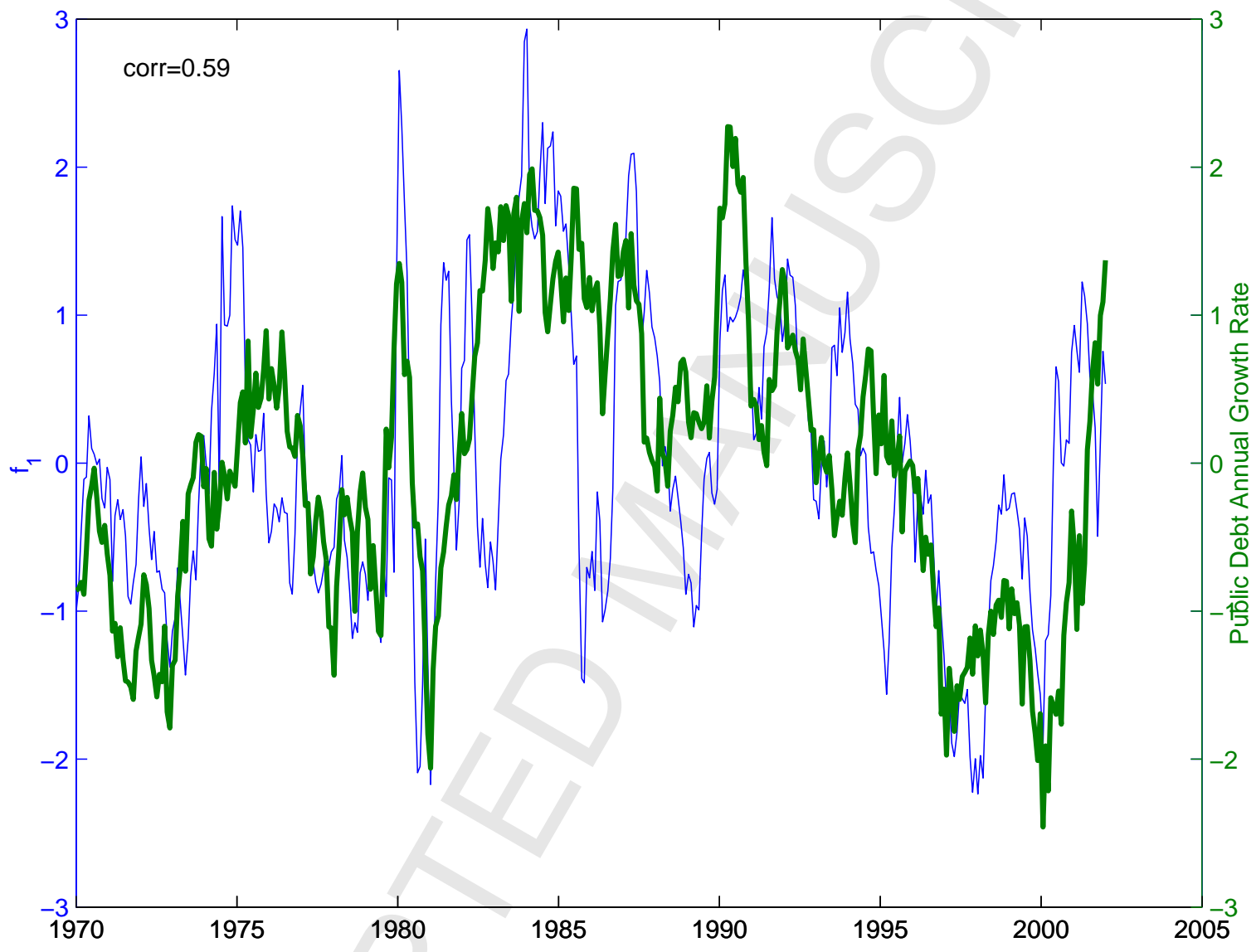

Fig. 5. The plot shows the monthly series of the estimated factor $f_{1}$ (thin line, left scale) against the annual government public debt growth rate (thick line, right scale). The latter series are residuals from regressing the debt growth rate on 12 lags of inflation and real activity. Both series are standardized to facilitate comparison. 

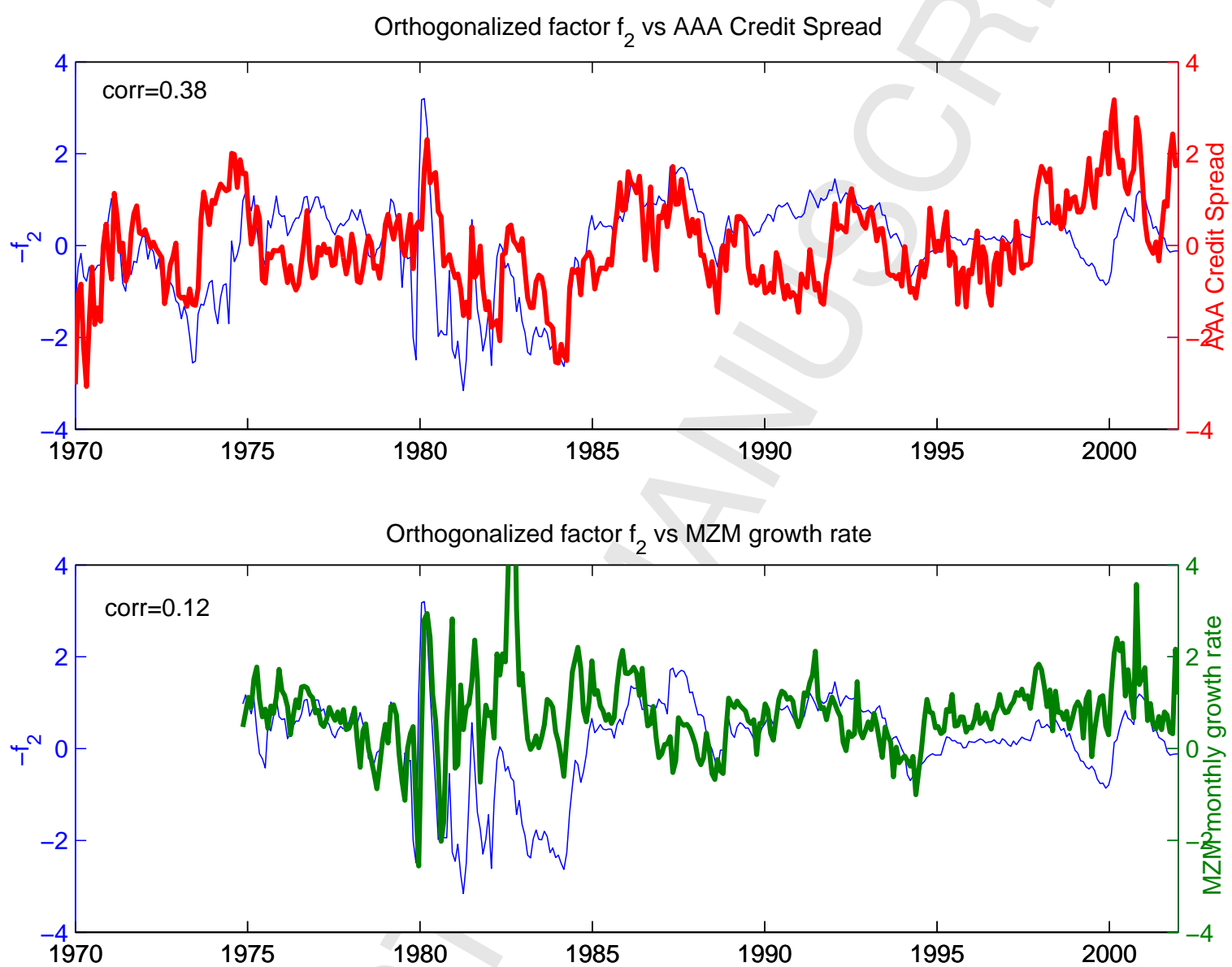

Fig. 6. The plot shows the monthly series of the estimated latent factor $f_{2}$ (with minus sign) (thin line, left scale) against the spread between AAA Moody's corporate index and the 10year Treasury bond yield in panel (a) and MZM monthly growth rate in panel (b) (thick line, right scale). Both macro series are residuals from regressing the AAA spread (or MZM rate) on inflation and real activity. All three series are standardized to facilitate comparison. The MZM series are available from 1975. 